Preprint typeset in JHEP style - HYPER VERSION

MIT-CTP-3717

hep-th/0602041

\title{
Brane Tilings and Exceptional Collections
}

\author{
Amihay Hanany ${ }^{1}$, Christopher P. Herzog ${ }^{2}$, David Vegh ${ }^{1}$ \\ ${ }^{1}$ Center for Theoretical Physics, Massachusetts Institute of Technology, \\ Cambridge, MA 02139, USA.* \\ ${ }^{2}$ Department of Phsyics, University of Washington, \\ Seattle, WA 98195, USA. ${ }^{\dagger}$ \\ hanany@mit.edu, herzog@u.washington.edu, dvegh@mit.edu
}

\begin{abstract}
:
Both brane tilings and exceptional collections are useful tools for describing the low energy gauge theory on a stack of D3-branes probing a Calabi-Yau singularity. We provide a dictionary that translates between these two heretofore unconnected languages. Given a brane tiling, we compute an exceptional collection of line bundles associated to the base of the non-compact Calabi-Yau threefold. Given an exceptional collection, we derive the periodic quiver of the gauge theory which is the graph theoretic dual of the brane tiling. Our results give new insight to the construction of quiver theories and their relation to geometry.
\end{abstract}

\footnotetext{
${ }^{*}$ Research supported in part by the CTP and the LNS of MIT and the U.S. Department of Energy under cooperative agreement \#DE-FC02-94ER40818. AH is also supported in part by the BSF American-Israeli Bi-National Science Foundation and a DOE OJI award.

${ }^{\dagger}$ Research supported in part by the U.S. Department of Energy under Grant No. DE-FG02-96ER40956.
} 


\section{Contents}

1. Introduction 1

2. Quivers and toric diagrams 3

3. Brane tilings 6

3.1 Tilings 6

3.2 Perfect matchings 9

3.3 Zig-zag and rhombus paths 11

4. Exceptional collections 13

4.1 From Exceptional Collection to Periodic Quiver 14

4.2 Line Bundles and Curvature Forms for Toric Surfaces 16

4.3 Bundles on $\mathbb{P}^{2} \quad 19$

4.4 Constructing the Quiver in General 20

4.5 Vanishing Euler Character 22

5. Compatibility 24

5.1 Beilinson quivers and internal matchings 24

5.2 Line bundles from tiling: The $\Psi$-map 26

5.3 Reconstructing the quiver 31

6. Conclusions 39

\section{Introduction}

Determining the low energy gauge theory on a stack of D-branes probing a Calabi-Yau singularity is an important, interesting, and in general unsolved problem. These D-brane constructions can be used to build flux vacua in string theory, and they play an important role in the AdS/CFT correspondence, where they yield a geometric understanding of strongly coupled gauge theories. While much progress has been made in understanding orbifold, toric, and other simple Calabi-Yau singularities, the general case remains elusive.

Two of the most powerful techniques for unearthing these gauge theories are the brane tiling method pioneered by [1, 2, 3] and exceptional collections first mentioned in the AdS/CFT context 
in [- The relation between these two methods has up to this point remained obscure. In this paper, we show how to translate one language into the other.

More specifically, we have in mind D3-branes in type IIB string theory. The ten dimensional geometry is divided up into a Minkowski part $\mathbb{R}^{3,1}$ which the D3-branes occupy and a transverse Calabi-Yau threefold $Y$. Placing the D3-branes at a singularity of $Y$ produces complicated quiver gauge theories which preserve $\mathcal{N}=1$ supersymmetry.

One of the best features of the brane tiling method is the ease with which the superpotential of the quiver gauge theory can be extracted. A brane tiling is a bipartite tiling of the torus $T^{2}$, and the superpotential terms are just the nodes of this tiling with coefficient \pm 1 given by the coloring of the node. No other method of relating gauge theory to geometric singularity has as yet produced such a simple way of extracting the superpotential.

For the brane tiling method to work, one starts with a toric Calabi-Yau three-fold singularity. The toric condition means that $Y$ possesses three $U(1)$ isometries. There are countably many interesting toric Calabi-Yau singularities, but the toric condition is a substantial restriction on $Y$. By using brane tilings, older algorithms ([5, 6]) get vastly simplified and reinterpreted.

For the exceptional collection method to work, one needs to be able to resolve partially the Calabi-Yau singularity by blowing up a complex surface - the exceptional collection lives on this surface. There are many both toric and non-toric Calabi-Yau singularities which can be resolved in this manner. The exceptional collection method was in large part developed to study some simple non-toric singularities, the non-toric del Pezzos [7].

While the superpotential can be extracted from an exceptional collection, the process is more abstract and less intuitive than for the brane tiling. In the exceptional collection case, deriving the superpotential requires working with $A$-infinity algebras [8, 9].

The exceptional collection method as applied to deriving quiver gauge theories rests on relatively firm mathematical and physical foundations [10, 11, 12, 13, 14]. From the perspective of the topological B-model, the objects in the collection can be understood as a nice basis of D-branes and the maps between the objects as massless open strings.

The brane tiling method began as an extremely remarkable observation: the tiling contains all the information of the quiver gauge theory, and hence proves to be a very useful tool in its study and construction. The toric diagram of the Calabi-Yau manifold can be easily obtained by either computing the determinant of the Kasteleyn matrix or by determining the zig-zag paths. Recent results 15] allow for computing the tiling directly from the toric diagram. More recently, the paper of [16] gave a physical interpretation of the dimer model as a tiling of D6-branes in the mirror topological A-model.

By providing a translation between the brane tiling and the exceptional collection, we put the brane tiling, along with its easy superpotential calculation, on a firmer mathematical and physical footing. Our results fall short of a general proof that the brane tiling method is equivalent to excep- 
tional collections for toric Calabi-Yau singularities. Instead, we provide a well motivated conjecture of the way this map will work which we can prove example by example. By relating the tiling to exceptional collections which are topological B-model objects, our approach is complementary to that of [16].

In order for our translation between the brane tiling and the exceptional collection to work, we henceforth restrict to toric Calabi-Yau threefold singularities which can be partially resolved by blowing up a complex surface.

In the next section, we begin by reviewing some elementary material about quivers, quiver gauge theories, and toric geometry. Section 3 contains a review of the brane tiling method. The principal results of the paper are contained in Sections 4 and 5.

Section 4 contains a brief review of the exceptional collection method and a map from the exceptional collection to the brane tiling. We argue that the periodic quiver which is the dual graph of the brane tiling can be constructed from a consideration of Wilson lines.

In Section 5, we proceed in the other direction, mapping the brane tiling onto an exceptional collection. The cornerstone of this mapping is the realization that internal perfect matchings are in one-to-one correspondence with exceptional collections of line bundles.

\section{Quivers and toric diagrams}

The matter content of the quiver gauge theory is neatly summarized in the quiver graph [17] which also generalizes the Dynkin diagrams. Each node in the quiver (see e.g. Figure 1) may carry an index, $N_{i}$, for the $i^{\text {th }}$ node and denotes a $U\left(N_{i}\right)$ gauge group. The edges (arrows) label the chiral bifundamental multiplets. These fields transform in the fundamental representation of $U\left(N_{i}\right)$ and in the anti-fundamental of $U\left(N_{j}\right)$ where $i$ and $j$ represent the nodes in the quiver that are the head and tail of the corresponding arrow.

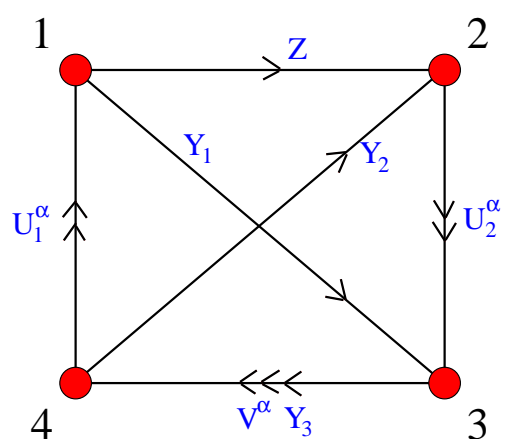

Figure 1: Quiver of $\mathbf{d} \mathbf{P}_{1}$. The theory contains four $U(N)$ gauge groups labeled by the nodes of the quiver. The arrows label bifundamental fields transforming in the (anti-)fundamental representation of the groups at the endpoints. 
To be gauge anomaly free, for each gauge group, the number of chiral fermions in the fundamental representation must equal the number in the antifundamental representation. This anomaly cancellation means that for a fixed node in the quiver, the number of incoming and outgoing arrows are the same.

By deleting certain arrows in the quiver, one obtains another graph, the so-called Beilinson quiver. In this quiver there exists an ordering of the nodes such that there are no arrows pointing backwards (for an example see Figure 2). Generically, there are many Beilinson quivers corresponding to a given quiver. These quivers can be thought of as subquivers that contain no oriented loops. A more precise definition can be found in Section 4 .

We are taking a small liberty with the term Beilinson quiver. Historically, Beilinson quiver referred only to projective space (see for example 18]). In the context of D-branes and Calabi-Yau manifolds, that would mean placing a stack of $\mathrm{D}$-branes at a singularity where a $\mathbb{P}^{2}$ had shrunk to zero size. The Beilinson quiver associated to $\mathbb{P}^{2}$ is then obtained by eliminating backward pointing arrows in the full gauge theory quiver. (These Beilinson quivers are sometimes called Bondal quivers [19.)

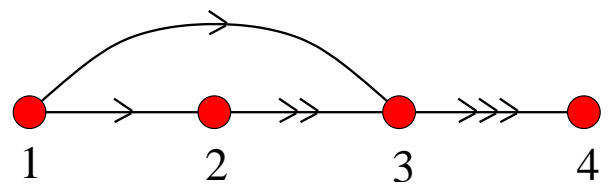

Figure 2: $\mathbf{d P}_{1}$ Beilinson quiver.

In order to fully specify the Lagrangian, we need to give the superpotential as well, which is a polynomial in gauge invariant operators. For example, for $\mathbf{d} \mathbf{P}_{1}$ the superpotential is

$$
W=\epsilon_{\alpha \beta} U_{1}^{\alpha} V^{\beta} Y_{1}-\epsilon_{\alpha \beta} U_{2}^{\alpha} Y_{2} V^{\beta}-\epsilon_{\alpha \beta} U_{1}^{\alpha} Y_{3} U_{2}^{\beta} Z .
$$

The AdS/CFT dual theory is determined by the Calabi-Yau threefold $Y$. For the purpose of this paper, we don't need explicit metrics. Instead, we will use toric geometry (20, 21]) to treat the topology of these singular manifolds. To use toric methods, we restrict the class of possible spaces to toric ones, i.e. the isometry group of $Y$ contains a 3 -torus. The variety can be defined by a strongly convex rational polyhedral cone $\sigma$ spanned by a set of vectors $\left(\left\{v_{r}\right\}\right)$ on the integer lattice $N$ (Figure 3).

The lattice is three dimensional so that we obtain a (complex) 3 d space. Let $M=\operatorname{Hom}(N, \mathbb{Z})$ be the dual lattice with pairing denoted by $\langle\cdot, \cdot\rangle$. The dual cone $\sigma^{v}$ is the set of vectors that are nonnegative on $\sigma$. The lattice points in $\sigma^{v}$ determine a finitely generated commutative semigroup:

$$
S_{\sigma}=\sigma^{v} \cap M=\{u \in M:\langle u, v\rangle \geq 0 \text { for all } v \in \sigma\} .
$$

The corresponding commutative $\mathbb{C}\left[S_{\sigma}\right]$ algebra defines the $U_{\sigma}$ variety by its spectrum

$$
U_{\sigma}=\operatorname{Spec}\left(\mathbb{C}\left[S_{\sigma}\right]\right) .
$$




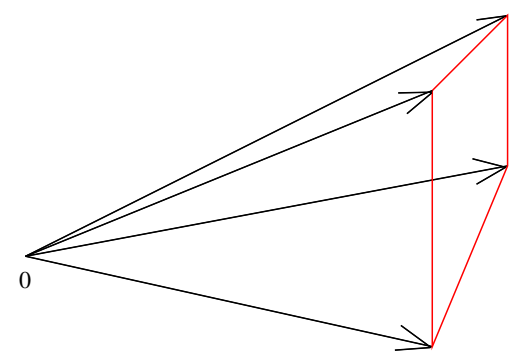

Figure 3: The cone for the variety. The coordinates of the spanning vectors are integers. The endpoints are coplanar following from the Calabi-Yau condition.

The so-called moment map for the torus action gives $Y$ as a Lagrangian $T^{3}$ fibration over the dual cone. For details of this map the reader should refer to [21].

For each spanning vector $v_{r}$ there is a corresponding $D_{r}$ (Weil) divisor in the toric variety. Principal divisors are of the form

$$
\sum_{r}\left\langle m, v_{r}\right\rangle D_{r}
$$

for $m \in M$. The Calabi-Yau condition states that $c_{1}(Y)=0$, i.e. the canonical class is trivial

$$
K=-\sum_{r} D_{r}=-\sum_{i}\left\langle m, v_{r}\right\rangle D_{r}
$$

The last equality implies that the endpoints of the $\left(\left\{v_{r}\right\}\right)$ vectors are coplanar, so with an appropriate $S L(3, \mathbb{Z})$ transformation a convex integer polygon in two dimensions can be obtained (see e.g. Figure (4). We will refer to this polygon as the toric diagram of the singularity [22, 23, 3]. Weil divisors can be specified as integer functions over the external lattice points of the toric diagram. Principal divisors are simply linear functions; the canonical class is a constant function.

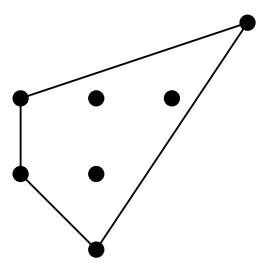

Figure 4: The toric diagram for $L^{1,7,3}$ which is part of the recently discovered series of $L^{a b c}$ metrics ( 24, 25]). The dual quiver theories have been constructed in [3, 26, 27. 


\section{Brane tilings}

\subsection{Tilings}

In this section we give a short introduction to brane tilings [2]. Brane tilings (a.k.a. dimer graphs) have arisen in two string theory contexts:

Quiver gauge theories that are obtained by placing D3-branes at the tip of a noncompact toric Calabi-Yau cone [1, 2, 15, 16], and

Topological string theory, more specifically, the partition function of the topological A-model defined on the same Calabi-Yau cone [28, 29].

In the following, we describe tilings from the first point of view. The brane tiling is a generalization of brane boxes [30, 31] and brane diamonds [32]. The tiling graph encodes the quiver and tree-level superpotential information, thus fully specifying the $4 \mathrm{~d} \mathcal{N}=1$ quiver theory Lagrangian. The toric diagram of the corresponding Calabi-Yau manifold can be easily computed by means of the Fast Forward Algorithm [2]. On the other hand, given the toric diagram, the tiling is simply obtained by the Fast Inverse Algorithm [15, 16]. The equivalence of the Forward and the Fast Forward Algorithms has recently been established in [33].

The brane tiling is a periodic bipartite graph $^{1}$ on the plane. Equivalently, one may draw it on the surface of a 2-torus. The faces label gauge groups, the edges are chiral bifundamental fields, and the nodes are terms in the superpotential. The dual graph of the brane tiling is the periodic quiver. Roughly speaking, the periodic quiver is the quiver drawn on a 2-torus such that the plaquettes give the terms in the superpotential. Figure 5 shows an example of a periodic quiver for the well known case of $\mathbb{C}^{3} / \mathbb{Z}_{3}$ (otherwise known as the complex cone over $\mathbb{P}^{2}$ ). Nodes carry three different labels and nodes with the same label are identified. The corresponding tiling is shown in Figure 6.

\begin{tabular}{c|c|c} 
Brane tiling & Periodic quiver & Gauge theory \\
\hline \hline faces & nodes & $U(N)$ gauge groups \\
edges & edges & bifundamental fields \\
nodes & plaquettes & superpotential terms
\end{tabular}

The tiling provides us with a simple geometrical unification of quiver and superpotential data. The bipartite property of the tiling implies that each face in the brane tiling has an even number of edges and that the dual quiver has an equal number of incoming and outgoing arrows for each gauge group. As discussed in Section 2, equal numbers of incoming and outgoing arrows are required by gauge anomaly cancellation. To each term in the superpotential there is a plaquette in the periodic

\footnotetext{
${ }^{1} \mathrm{~A}$ planar graph is bipartite if the nodes can be colored in black and white, such that edges only connect black nodes to white nodes and vice versa.
} 
quiver and a black or white node in the tiling. The color of the node in the tiling tells us the sign of the term. Since a bifundamental field joins a white and black node in the tiling, we conclude that each bifundamental field appears exactly twice in the superpotential, once with a plus and once with a minus sign.

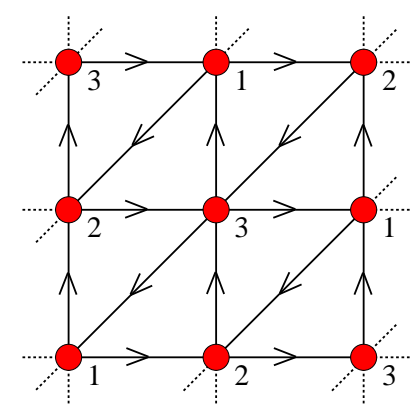

Figure 5: The $\mathbb{P}^{2}$ periodic quiver. The nodes denote $U(N)$ gauge groups; the directed edges between them are bifundamental fields. The plaquettes of the quiver graph are terms in the superpotential. This example has three gauge groups, labeled by numbers. Identifying nodes with the same labels (i.e. "compactifying" the periodic quiver) yields the usual quiver diagram.
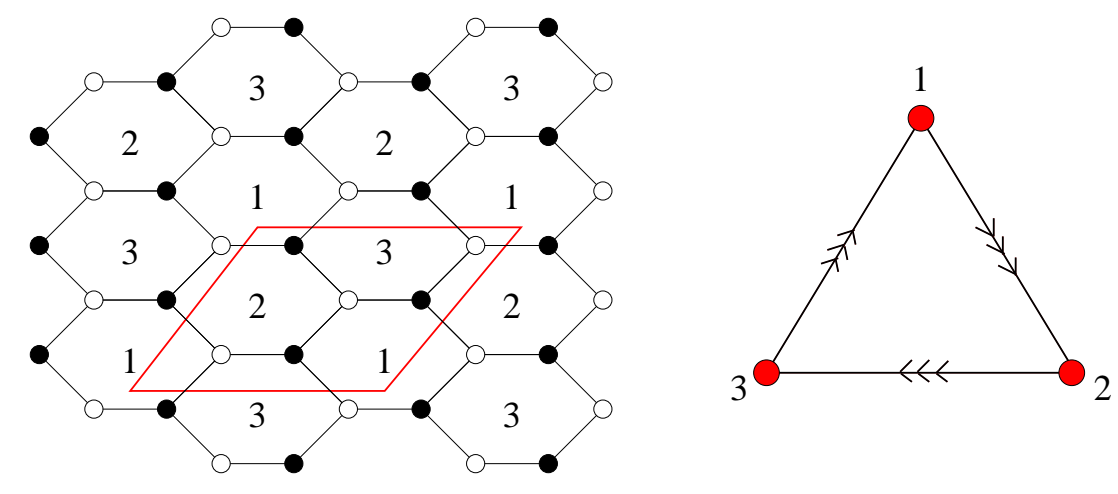

Figure 6: $\mathbb{P}^{2}$ brane tiling and quiver. The unit cell of the lattice is shown in red. The theory has three gauge groups (faces in the tiling) and six cubic terms in the superpotential (valence three nodes of the tiling).

As a simple example, Figure 6 shows the brane tiling and the quiver for $\mathbb{P}^{2}$. We see that the brane tiling contains three faces; these correspond to the three gauge groups (nodes) in the quiver. The nine edges in the tiling are the bifundamental fields. The six nodes of the tiling immediately give the following superpotential:

$$
\begin{gathered}
W=X_{12}^{(1)} X_{23}^{(2)} X_{31}^{(3)}+X_{12}^{(2)} X_{23}^{(3)} X_{31}^{(1)}+X_{12}^{(3)} X_{23}^{(1)} X_{31}^{(2)} \\
-X_{12}^{(3)} X_{23}^{(2)} X_{31}^{(1)}-X_{12}^{(2)} X_{23}^{(1)} X_{31}^{(3)}-X_{12}^{(1)} X_{23}^{(3)} X_{31}^{(2)}
\end{gathered}
$$


Here $X_{i j}^{(k)}$ denotes the bifundamentals going from gauge group $i$ to $j$, and $k$ labels the different fields.

Another example is the del Pezzo $1\left(\mathbf{d P}_{\mathbf{1}}\right)$ theory (Figure 7). The tiling contains four faces which label the four gauge groups.

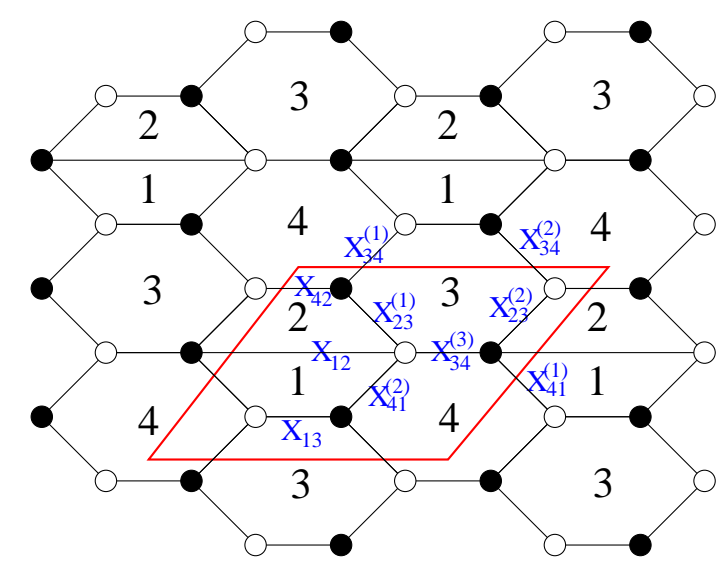

Figure 7: Brane tiling for $\mathbf{d P}_{1}$. The fundamental cell of the periodic graph is shown in red, the fields corresponding to the edges are shown in blue. The numbers label the faces that correspond to the groups (nodes) in the dual quiver graph.

We have already seen the corresponding quiver (Figure 1). The tiling gives the superpotential:

$$
\begin{aligned}
W & =X_{23}^{(1)} X_{34}^{(1)} X_{42}+X_{12} X_{23}^{(2)} X_{34}^{(3)} X_{41}^{(1)}+X_{13} X_{34}^{(2)} X_{41}^{(2)} \\
& -X_{13} X_{34}^{(1)} X_{41}^{(1)}-X_{12} X_{23}^{(1)} X_{34}^{(3)} X_{41}^{(2)}-X_{23}^{(2)} X_{34}^{(2)} X_{42}
\end{aligned}
$$

which, after making the relabeling $\left\{X_{42}=Y_{2}, X_{12}=Z, X_{13}=Y_{1}, X_{34}^{(\alpha)}=V^{(\alpha)}, X_{34}^{(3)}=\right.$ $\left.Y_{3}, X_{23}^{(\alpha)}=U_{2}^{(2-\alpha)}, \quad X_{41}^{(\alpha)}=U_{1}^{(2-\alpha)}\right\}$, is the same as 2.1).

One can compute the toric diagram related to the moduli space of this theory by means of the Kasteleyn matrix (3.3). The Kasteleyn matrix is the adjacency matrix of the tiling graph. More precisely, the rows are labeled by the black nodes, the columns by the white nodes. The corresponding entry is zero if the two nodes are not connected; otherwise it is the appropriate weight of the connecting edge. For details of building the Kasteleyn matrix the reader should refer to [2, 1, 34, 35].

$$
K=\left(\begin{array}{ccc}
z^{-1} & 1 & w^{-1} \\
1 & 1-z & z \\
w & 1 & 1
\end{array}\right)
$$

The determinant of the Kasteleyn matrix gives the spectral curve

$$
P(w, z) \equiv \operatorname{det} K=-4+w^{-1}+z^{-1}+z+w z
$$


The $P(w, z)=x y$ equation and its deformations describe the mirror Calabi-Yau as a fibration. The Newton polygon of this polynomial gives the toric diagram of the threefold (Figure 8) ${ }^{2}$

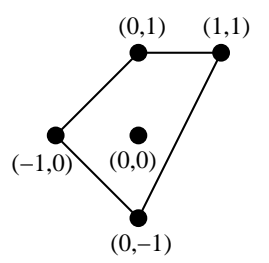

Figure 8: Toric diagram for $\mathbf{d} \mathbf{P}_{1}$. The multiplicity of the internal point is four.

\subsection{Perfect matchings}

A perfect matching is a subgraph of the tiling that contains all the nodes and each node has valence one [35, 34]. This means that a perfect matching is a set of dimers (edges in the brane tiling) that are separated, i.e. they don't touch each other; furthermore the dimers cover all the nodes. Therefore, we have altogether $V / 2$ dimers in each perfect matching, where $V$ denotes the number of nodes in the tiling. The eight perfect matchings for $\mathbf{d P}_{1}$ are shown in Figure 9 .

It can be easily checked that if we superimpose two perfect matchings $A$ and $B$ (denoted $A+B$ ), then we obtain loops (and separate edges which we neglect). Fix a reference perfect matching $R$. For each matching $A_{i}$ we can define an integer height function. The loops of $R+A_{i}$ can be regarded as "contours". Crossing a loop at an edge where the black node is on the left hand side means a change in the height function by \pm 1 . The sign depends on whether the edge was part of the reference matching $(-1)$ or that of $A_{i}(+1)$. An example is shown in Figure 10 which is the height function for $\mathbf{d P}_{1}$ for the last matching in Figure 9. The shading indicates the height. The contours are made of blue and green edges that are contained in the last matching in the list and in the reference matching (4th matching in the list), respectively.

The above defined height function is a well-defined function on the infinite periodic tiling faces, but on the torus it has monodromy that is described by two integers: $(s, t) .{ }^{3}$ For our example this pair was $(0,1)$. Such pairs are assigned to every perfect matching with respect to a reference matching. These pairs are coordinates of points in the toric diagram; in fact, the toric diagram is the (convex) set of all such points. The reference matching has $(0,0)$ coordinates and the change in the reference matching merely translates the toric diagram. An $S L(2, \mathbb{Z})$ transformed fundamental cell results in an $S L(2, \mathbb{Z})$ transformed toric diagram. Perfect matchings that reside at an internal lattice point in the toric diagram are called internal matchings. The remaining matchings at the external points are the external matchings with corresponding Weil divisors as discussed in section 2 .

\footnotetext{
${ }^{2}$ The toric diagram can also be computed by means of the zig-zag paths of the tiling; for details, see 15.

${ }^{3}(s, t)$ denotes the change in the height as we go along the two non-trivial cycles of the torus of the brane tiling. This pair is also known as the slope of the height function.
} 

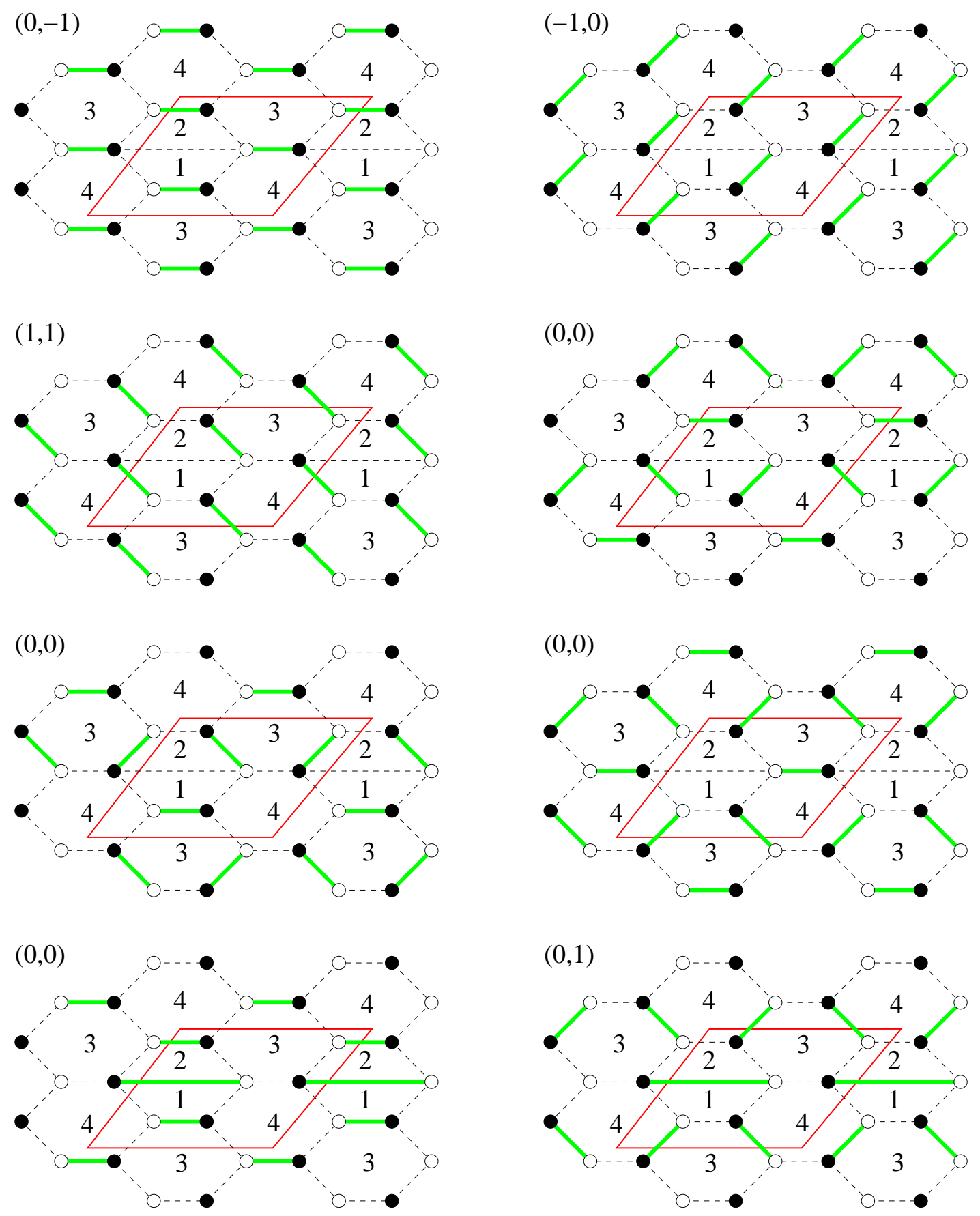

Figure 9: The eight periodic perfect matchings of $\mathbf{d P}_{1}$. The green edges are contained in the matching. The dashed lines are the edges left in the tiling. The $(s, t)$ numbers are the corresponding points in the toric diagram (see Figure 8). 


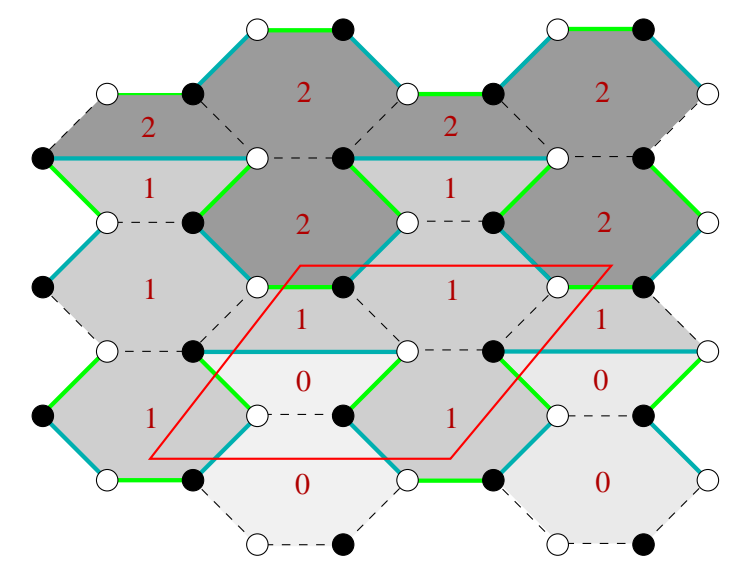

Figure 10: The height function for the last perfect matching of $\mathbf{d P}_{1}$. The edges in the matching are colored blue. The green reference matching was chosen to be the $4^{\text {th }}$ perfect matching from Figure 9 . The two matchings on top of each other result in horizontal loops where the height function increases by one. The monodromy of the height function is $(0,1)$ and there is a corresponding lattice point in the toric diagram in Figure 8.

\subsection{Zig-zag and rhombus paths}

In [15] a special path was defined which turns out to be also useful here. A zig-zag path is a path on the edges of the tiling which turns maximally left at a node, then maximally right at the next node, then again left, and so on [34]. An example is presented in Figure 11.

In 15] it was an observation that these loops on the torus of the tiling are in one-to-one correspondence with the edges of the toric diagram polygon. In fact, their homology classes give the outward pointing normal vectors of the edges, the so-called external pq-legs. This follows from the results of $\S 7.1$ in 16$]$.

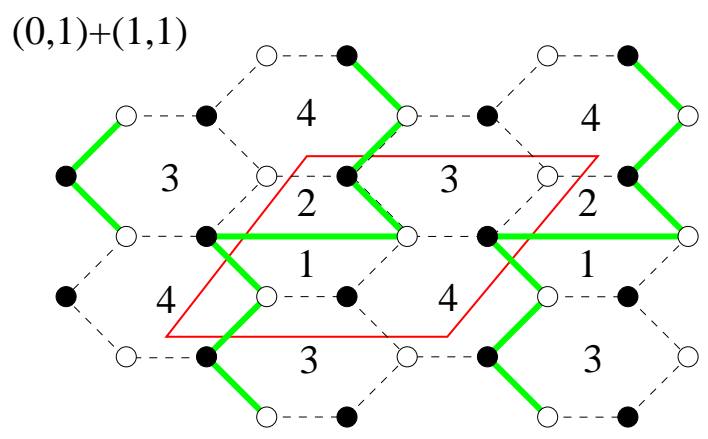

Figure 11: Zig-zag path in the $\mathbf{d P}_{\mathbf{1}}$ brane tiling. The path is the superposition of the $(0,1)$ and $(1,1)$ neighboring external perfect matchings.

As discussed at length in [15], one can associate to the brane tiling another graph, which 
contains the edges that connect the face centers to the tiling nodes. We call this graph the rhombus lattice. ${ }^{4}$ The zig-zags in the tiling are "straight" rhombus paths in the rhombus lattice (Figure 12).
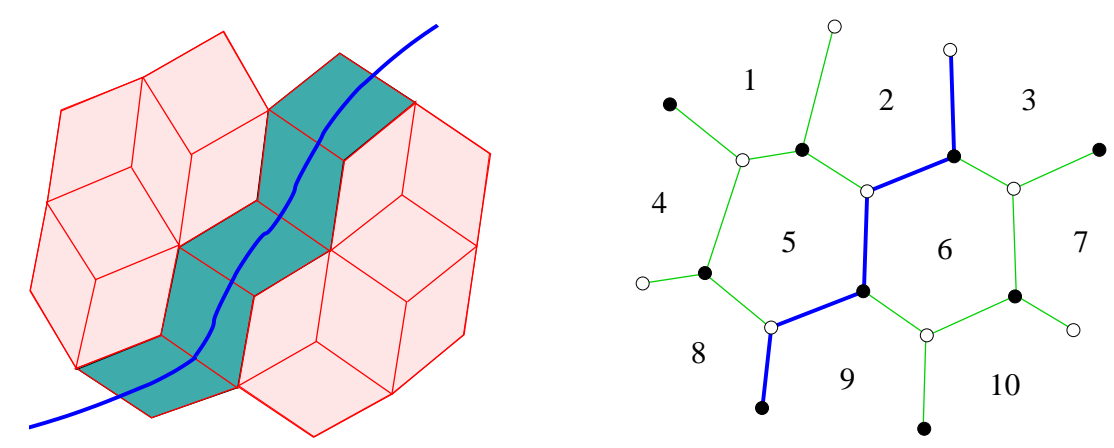

Figure 12: (i) Rhombus path in the rhombus lattice. (ii) Equivalent zig-zag path in the brane tiling. The blue line shows the rhombus loop schematically. The edges which are crossed by the blue line in (i) are all parallel. Their orientation can be described by an angle, the so-called rhombus loop angle.

There are always two zig-zag paths going through each tiling edge. In the rhombus lattice this translates to the fact that the bifundamentals arise from the intersection of two rhombus paths. These paths have been analyzed in considerable detail in [16] where the zig-zags were related to cycles that are wrapped by D6-branes in the mirror Calabi-Yau. Further developments in brane tilings which will not be discussed here can be found in [36, 37].

\footnotetext{
${ }^{4}$ The faces of this new graph are indeed rhombi if the original brane tiling is isoradially embedded in the plane. For details see 15 .
} 


\section{Exceptional collections}

Exceptional collections provide a powerful tool for deriving the low energy gauge theory description of a stack of D-branes probing a Calabi-Yau singularity. Given a Calabi-Yau cone $Y$, a stack of Dbranes at the singularity will fragment into a set of fractional branes from which the gauge theory is easily deduced. These fractional branes are best described as objects in $D^{b}(Y)$, the derived category of coherent sheaves on $Y$. Exceptional collections provide a way of finding a good set of fractional branes and avoiding a direct confrontation with $D^{b}(Y) .^{5}$

If $Y$ can be partially resolved by blowing up a possibly singular complex surface $V$, instead of looking for fractional branes on $Y$, we look for an exceptional collection of sheaves on $V$. There is then a simple procedure for converting this collection into a good set of fractional branes [10, 11], and in fact the gauge theory can often be deduced directly from the exceptional collection.

An exceptional collection of sheaves $\mathcal{E}=\left(E_{1}, E_{2}, \ldots, E_{n}\right)$ is an ordered set of sheaves which satisfy the following special properties:

1. Each $E_{i}$ is exceptional: $\operatorname{Ext}^{q}\left(E_{i}, E_{i}\right)=0$ for $q>0$ and $\operatorname{Ext}^{0}\left(E_{i}, E_{i}\right)=\operatorname{Hom}\left(E_{i}, E_{i}\right)=\mathbb{C}$.

2. $\operatorname{Ext}^{q}\left(E_{i}, E_{j}\right)=0$ for $i>j$ and $\forall q$.

In these notes, we will be most interested in the case where the collection is strongly exceptional, in which case $\operatorname{Ext}^{q}\left(E_{i}, E_{j}\right)=0$ for $i<j$ and $q>0$. For smooth toric surfaces, the collection must be strong to generate a physical quiver gauge theory [10, 11], and the same is true for singular surfaces as well. ${ }^{6}$

For the most part, our sheaves can be thought of as line bundles, and line bundles are easy to describe in a toric context. ${ }^{7}$ For each ray $v_{r}$ in the fan, there is a toric Weil divisor $D_{r}$. The line bundles can then be expressed as $\mathcal{O}\left(\sum_{r} a_{r} D_{r}\right)$ for $a_{r} \in \mathbb{Z}$. One very special line bundle is the anti-canonical bundle:

$$
\mathcal{O}(-K)=\mathcal{O}\left(\sum_{r} D_{r}\right) .
$$

As we said earlier, the Calabi-Yau cone is the total space of the canonical bundle over our surface. The fact that our fan defines a convex polygon means that $K$ is negative.

Given a strongly exceptional collection $\mathcal{E}$, the quiver gauge theory can be constructed from the inverse collection $\mathcal{E}^{\vee}$. The members of $\mathcal{E}^{\vee}$ are no longer sheaves but objects in $D^{b}(V)$. Lifting these objects to $Y$ yields the fractional branes. At the level of D-brane charges, the inverse collection can be constructed from the Euler character on $V, \chi\left(E_{i}, E_{j}^{\vee}\right)=\delta_{i j}$. As a set of objects in $D^{b}(V), \mathcal{E}^{\vee}$ is constructed via a braiding operation called mutation described in detail in 10. The

\footnotetext{
${ }^{5}$ For earlier physics applications of exceptional collections to Landau Ginzburg models, see [38, 39, 40, 41, 42.

${ }^{6}$ For a recent gauge theory interpretation of more general exceptional collections, see [43].

${ }^{7}$ For singular surfaces when $D$ is not a Cartier divisor, $\mathcal{O}(D)$ is actually not a line bundle but only a reflexive sheaf. Nevertheless, for simplicity, we will not emphasize this point further.
} 
inverse collection is also exceptional although no longer strongly exceptional. The Euler character $\chi\left(E_{i}^{\vee}, E_{j}^{\vee}\right)$ can be interpreted as the number of arrows in the quiver from node $i$ to node $j$ minus the number of arrows from node $j$ to node $i$ [14, 11]. This matrix is sometimes referred to as the antisymmetric part of the adjacency matrix. More precisely, the Euler character tells us the net number of $\operatorname{Hom}_{D^{b}(Y)}^{1}\left(E_{i}^{\vee}, E_{j}^{\vee}\right)$ maps in the Calabi-Yau between the fractional branes. For each of these maps, we have a massless open string which translates into a bifundamental field in the quiver gauge theory.

It is often convenient to write down an intermediate quiver, the so-called Beilinson quiver, which lives on $V$ instead of $Y$. This quiver contains arrows corresponding only to the negative entries of $\chi\left(E_{i}^{\vee}, E_{j}^{\vee}\right)$, or more precisely maps in $\operatorname{Ext}^{1}\left(E_{i}^{\vee}, E_{j}^{\vee}\right)$. The Beilinson quiver algebra can be thought of as

$$
\oplus_{i, j} \operatorname{Hom}\left(E_{i}, E_{j}\right)
$$

but the quiver contains arrows only for the generators of this algebra which are encoded simply in $\mathcal{E}^{\vee}$. Because $V$ is compact, the Beilinson quiver contains no oriented loops.

\subsection{From Exceptional Collection to Periodic Quiver}

In this section we assume that we have a compact toric surface $V$ with positive anti-canonical class and a strongly exceptional collection of line bundles $\mathcal{E}$ on $V$. We would like to construct from this data a periodic quiver. In particular, we will write the Beilinson quiver on a torus.

Any toric surface can be described by a fan by which we mean a collection of at least three vectors $v_{r}, r=1, \ldots, n$ on an integer lattice $\mathbb{Z}^{2}$. That the surface is compact means that the polygon defined by the endpoints of the vectors $v_{r}$ includes the origin. That the anti-canonical class of this surface is positive means that the polygon is convex. (We would like to allow $V$ to have quotient singularities.)

One way of understanding $V$ is as a quotient of $\mathbb{C}^{n}$. Given $n$ vectors in $\mathbb{Z}^{2}$, we expect that there will be $n-2$ linearly independent relations between the $v_{r}$, which we write as

$$
\sum_{r} Q_{a r} v_{r}=0
$$

where $a=1, \ldots, n-2$ and $Q_{a r} \in \mathbb{Z}$. Geometrically, we quotient

$$
\frac{\mathbb{C}^{n}-F_{\Delta}}{\left(\mathbb{C}^{*}\right)^{n-2}}
$$

where the action of the $\left(\mathbb{C}^{*}\right)^{n-2}$ is given by the $Q_{a r}$. The set $F_{\Delta}$ is a small set of points inside $\mathbb{C}^{n}$ which we need to remove to have a well defined quotient.

As an example, consider $\mathbb{P}^{2}$ for which the fan is $v_{1}=(1,0), v_{2}=(0,1)$, and $v_{3}=(-1,-1)$. There is just one relation which we write as $Q=(1,1,1)$. This quotient construction is nothing but the usual equivalence relation of the homogenous coordinates on $\mathbb{P}^{2}$, namely $\left(X_{1}, X_{2}, X_{3}\right) \sim$ $\left(\lambda X_{1}, \lambda X_{2}, \lambda X_{3}\right)$ for $\lambda \in \mathbb{C}^{*} . F_{\Delta}$ is the origin $(0,0,0)$ of $\mathbb{C}^{3}$. 
For arbitrary $V$, we can think of $X \in \mathbb{C}^{n}$ as generalized homogenous coordinates. The $n-2$ equivalence relations (4.3) leave a two complex dimensional space which is $V$ itself:

$$
\left(X_{1}, X_{2}, \ldots, X_{n}\right) \sim\left(\lambda^{Q_{a 1}} X_{1}, \lambda^{Q_{a 2}} X_{2}, \ldots, \lambda^{Q_{a n}} X_{n}\right) .
$$

This two complex dimensional space $V$ is a fiber bundle $\pi: V \rightarrow B$ where $B$ is a real two dimensional surface and the fibers are real, two dimensional tori. More simply put, the fibers are coordinatized by the phase angles of the complex coordinates on $V$. First, we characterize this torus in greater detail.

Given the $n-2$ vectors $Q_{a}$ and using the standard inner product on $\mathbb{Z}^{n}$, we find two additional vectors $q_{1}$ and $q_{2}$ such that $q_{i} \cdot Q_{a}=0$ and $q_{1}$ and $q_{2}$ are linearly independent. A canonical set of $q_{i}$ are the $v_{r}$ reinterpreted as two $n$ dimensional vectors rather than $n$ two dimensional vectors: we could set $q_{1 r}=v_{r, 1}$ and $q_{2 r}=v_{r, 2}$. These $q_{i}$ can be used to measure relative positions on the real two torus. Given the homogenous coordinates $\left(X_{1}, X_{2}, \ldots, X_{n}\right)$, we define the two torus coordinates to be

$$
\left(\theta_{1}, \theta_{2}\right)=\left(\sum_{r} q_{1 r} \operatorname{Arg} X_{r}, \sum_{r} q_{2 r} \operatorname{Arg} X_{r}\right)
$$

Notice that if we shift $X_{r}$ by $\lambda^{Q_{a r}},\left(\theta_{1}, \theta_{2}\right)$ remains invariant because $q_{i} \cdot Q_{a}=0$.

Our D-branes are line bundles on $V$, and thus we can think of them as Euclidean D4-branes filling all of $V$. If we perform fiberwise $\mathrm{T}$-duality twice on the two torus, we should find D2-branes localized at points on the torus. The open strings will then connect these points together. The periodic Beilinson quiver is nothing but this web of D2-branes and open strings.

We will characterize this web using the original line bundle (or D4-brane) description. The notation $\mathcal{O}(D)$ indicates a D4-brane with a dissolved D2-brane; this dissolved D2-brane has the same charges as a D2-brane wrapping the divisor $D \subset V$. We can describe this dissolved D2brane as magnetic flux. Because the line bundle is holomorphic, the field strength components $F_{i j}=0=F_{\bar{\imath} \bar{\jmath}}$ vanish, and locally the field strength takes the form

$$
F=i \partial_{i} \bar{\partial}_{\bar{\jmath}}\left(f+f^{*}\right) d y^{i} \wedge d \bar{y}^{\bar{\jmath}}
$$

where $A_{j}=-i \partial_{j} f, A_{\bar{\jmath}}=i \bar{\partial}_{\bar{\jmath}} f^{*}$ and $f$ is some function of the coordinate patch. By a gauge choice, we may take the imaginary part of $f$ to vanish.

In a toric variety, the phase angle directions $\theta_{i}$ are isometries, and the field strength $F$ describing the D2-brane should not depend on the $\theta_{i}$. Because our variety is toric, we can choose a complex structure such that $y^{j}=\ln r_{j}+i \theta_{j}=\rho_{j}+i \theta_{j}$. In this coordinate system, the field strength becomes

$$
F=\left(\frac{\partial^{2} f}{\partial \rho_{i} \partial \rho_{j}}+\frac{\partial^{2} f}{\partial \theta_{i} \partial \theta_{j}}\right) d \rho_{i} \wedge d \theta_{j}+\frac{\partial^{2} f}{\partial \theta_{i} \partial \rho_{j}}\left(d \rho_{i} \wedge d \rho_{j}+d \theta_{i} \wedge d \theta_{j}\right) .
$$

In order for $F$ to be independent of $\theta_{i}, f$ must take a very special form. In particular, $f=$ $g(r)+C_{i \bar{\jmath}} y^{i} \bar{y}^{\bar{\jmath}}$ where the second term leads to a constant field strength. We will assume this second 
term in $f$ vanishes in which case the vector potential takes the very simple form

$$
A=\frac{\partial f}{\partial \rho_{i}} d \theta_{i}
$$

At this point, we fix a point $\left(r_{1}, r_{2}\right) \in B$ and look at the $T^{2}$ fiber, where we recognize a Wilson line. Locally on the $T^{2}, A=w_{j} d \theta_{j}$ is pure gauge; $A=i d \ln \Lambda$ where $\Lambda=\exp \left(-i w_{j} \theta_{j}\right)$. However, globally, $\Lambda$ does not respect the periodicity conditions. We have a distinct set of Wilson lines for $0 \leq w_{j}<1$, with $\left(w_{1}, w_{2}\right) \sim\left(w_{1}+n, w_{2}+m\right)$ for $n$ and $m$ integers. This set of Wilson lines lives on a dual torus we will call $\tilde{T}^{2}$.

Given a collection of line bundles, we can calculate the value of the Wilson line for each such bundle and plot that point $\left(w_{1}, w_{2}\right)$ on our $\tilde{T}^{2}$ of length and height one. This plot gives us the nodes of the periodic Beilinson quiver.

The strings between the D4-branes come from the generators of the Beilinson quiver algebra and as such are maps of the form $\operatorname{Hom}\left(E_{i}, E_{j}\right)$. Since the branes are line bundles, we may write $E_{i}=\mathcal{O}(D), E_{j}=\mathcal{O}\left(D^{\prime}\right)$, and $\operatorname{Hom}\left(E_{i}, E_{j}\right)=H^{0}\left(V, \mathcal{O}\left(D^{\prime}-D\right)\right)$. We expect, given a generating element in $\operatorname{Hom}\left(E_{i}, E_{j}\right)$, to find a corresponding string between $\mathcal{O}(D)$ and $\mathcal{O}\left(D^{\prime}\right)$. Moreover, $\mathcal{O}(D)$ and $\mathcal{O}\left(D^{\prime}\right)$ should be separated by a vector on the torus given by the value of the Wilson line for $\mathcal{O}\left(D^{\prime}-D\right)$.

From the derived category point of view on $Y$, we know how to compute the masses of these open strings 10, 11, 44, and the answer depends on being able to understand instanton corrections as we move in the Kähler moduli space of $Y$. From the point of view of the complex surface $V$ and the Wilson line discussion, our intuition is that a string stretching between two of these D4-branes

will have a mass proportional to the distance between the corresponding points on $\tilde{T}^{2}$ 45. As we change the base point, the Wilson lines will all move around. Our naive expectation is that for massless strings, there is a particular choice of base point for which the Wilson line corresponding to $\mathcal{O}\left(D^{\prime}-D\right)$ vanishes. It would be interesting to understand these masses better from the Wilson line point of view.

\subsection{Line Bundles and Curvature Forms for Toric Surfaces}

In the previous section, we sketched a procedure for converting a set of line bundles on a toric variety into a periodic quiver, but we did not explain why the construction would respect the periodicity of the torus. For example, take two linearly equivalent divisors $D$ and $D^{\prime}$. The corresponding line bundles $\mathcal{O}(D)$ and $\mathcal{O}\left(D^{\prime}\right)$ correspond to the same D-brane. Why then are the Wilson lines for $\mathcal{O}(D)$ and $\mathcal{O}\left(D^{\prime}\right)$ the same? In this section, we will attempt to answer this question and elucidate the structure of the corresponding vector potentials.

Given a line bundle, $\mathcal{O}(D)$, and a particular choice of Kähler metric on a toric variety, one can construct an explicit coordinate dependent expression for a representative of $c_{1}(D) \in H^{2}(V, \mathbb{Z})$. These representatives were first worked out by 46] (for a readable and more recent account see 447). 
This representative of $\mathcal{O}(D)$ is holomorphic, i.e. locally of the form $i \partial \bar{\partial} f$. Also, it is independent of the angular coordinates $\theta_{i}$ and so takes the form (4.9) discussed previously.

These representatives have a number of disadvantages. In most cases, these representatives do not satisfy the remaining equation of motion $g^{i \bar{\jmath}} F_{i \bar{\jmath}}=\mu$. Here, $\mu$ is a constant often called the slope. Moreover, they depend on a particular canonical choice of Kähler metric which is usually not the one of physical interest. Typically, we would be more interested in a metric which is compatible with a Ricci flat metric on the cone over $V .{ }^{8}$ Despite these disadvantages, we use these explicit representatives for they form a useful beginning from which to argue more general results.

We have thus far been working with complex coordinates $\rho+i \theta$, but these representatives are most easily expressed in symplectic coordinates on $V, x+i \theta$. The phase angles $\theta_{i}$ remain the same in both the complex and symplectic system. For the $x$, we define a polytope

$$
\Delta=\left\{x \in \mathbb{R}^{2}:\left\langle x, v_{r}\right\rangle \geq-1 \forall r\right\}
$$

The symplectic form is then $\omega=\sum_{i} d x_{i} \wedge d \theta_{i}$.

In these symplectic coordinates, the Kähler metric and complex structure depend on a potential function $g(x)$. Define

$$
g_{i j}=\frac{\partial^{2} g(x)}{\partial x_{i} \partial x_{j}}
$$

The line element becomes

$$
d s^{2}=g_{i j} d x_{i} d x_{j}+g^{i j} d \theta_{i} d \theta_{j}
$$

where $g^{i j}$ is the inverse of $g_{i j}$ and summation on the indices is implied. The symplectic coordinates are related to the complex ones by a Legendre transformation, $\rho=\partial g / \partial x$.

The representatives of $H^{2}(V, \mathbb{Z})$ depend on a particular choice of $g$,

$$
g_{\text {can }}=\frac{1}{2} \sum_{r} \ell_{r} \log \ell_{r}
$$

where we have defined

$$
\ell_{r}=\left\langle x, v_{r}\right\rangle+1
$$

In the case of projective space, this metric is physically interesting: it's Einstein and is thus compatible with a Ricci flat metric on the cone over $V$. In general $g_{\text {can }}$ will produce a metric which is physically uninteresting albeit simple. A general Kähler metric is related to $g_{c a n}$ in a smooth way:

$$
g=g_{\text {can }}+h
$$

where $h$ is a smooth function on $\Delta$.

\footnotetext{
${ }^{8}$ It may be that the metric compatible with a Ricci flat metric on the cone is not Kähler. For example, the metric on $\mathbf{d} \mathbf{P}_{1}$ compatible with the $Y^{2,1}$ Sasaki-Einstein metric is not Kähler [22].
} 
We have seen already that a holomorphic vector bundle has a curvature form which may be written as $2 i \partial \bar{\partial} f(\rho)$ for some locally defined function of $f$. In symplectic coordinates, this two-form becomes

$$
2 i \partial \bar{\partial} f=\sum_{j, k} \frac{\partial}{\partial x_{j}}\left(g^{k l} \frac{\partial f}{\partial x_{l}}\right) d x_{j} \wedge d \theta_{k} .
$$

For the canonical choice of metric, we take the vector potential corresponding to $\mathcal{O}\left(D_{r}\right)$ to be

$$
A_{r}=\frac{1}{2}\left(g_{c a n}\right)^{k l} \frac{\partial \log \ell_{r}}{\partial x_{l}} d \theta_{k}
$$

This $A_{r}$ yields a curvature two-form which represents the class $c_{1}\left(D_{r}\right)$ but is in general not harmonic. Note that $A_{r}$ is only well defined away from the side $\ell_{r}=0$.

Using (4.17), we will prove a result about the $A_{r}$ and then argue that the same result must hold more generally for non-canonical metrics and $A_{r}$ which do satisfy the equations of motion. The result is that

$$
\sum_{r} v_{r, i} A_{r}=d \theta_{i}
$$

or in other words, this particular combination of the $A_{r}$ is pure gauge. The result follows simply from noting that

$$
\left(g_{c a n}\right)_{i j}=\sum_{r} \frac{v_{r, i} v_{r, j}}{2 \ell_{r}}
$$

More generally, because every divisor $D=\sum_{r} a_{r} D_{r}$ can be expressed as a sum of primitive Weil divisors, we expect there to be a basis of primitive vector potentials $A_{r}, r=1, \ldots, n$ such that $A_{D}=\sum_{r} a_{r} A_{r}$. We have now chosen the $A_{r}$ to satisfy the equations of motion, but they should be related to the canonical $A_{r}$ in a smooth way. We say two divisors $D$ and $D^{\prime}$ are linearly equivalent when they have the same $Q$ charges, $\sum_{r} Q_{a r}\left(a_{r}-a_{r}^{\prime}\right)=0$. All such linear equivalence relations are generated by the $q_{i}$. If $D$ and $D^{\prime}$ are linearly equivalent, then $\mathcal{O}\left(D-D^{\prime}\right) \sim \mathcal{O}$. But $\mathcal{O}$ corresponds to a single D4-brane with no dissolved D2-brane charge. The associated field strength must vanish, and it must be that

$$
\sum_{r} q_{i r} A_{r}
$$

is pure gauge for $i=1$ and 2 .

We can deduce more from the statement that (4.20) is pure gauge. A gauge transformation $A \rightarrow A+i d \ln \Lambda$ must respect the periodicity of the torus. Since the $A_{r}$ take the form $f(r) d \theta$, the gauge transformation $\ln \Lambda$ which annihilates (4.20) must depend only linearly on $\theta$ and not at all on $x$. The only choice is $\Lambda=\exp (\operatorname{in} \theta)$, from which we conclude that

$$
\sum_{r} q_{i r} A_{r}=n_{i 1} d \theta_{1}+n_{i 2} d \theta_{2}
$$


for integers $n_{i j}$. The $v_{r}$ and our Wilson line torus are only defined up to an $S L_{2}(\mathbb{Z})$ transformation so we choose

$$
\sum_{r} q_{1 r} A_{r}=d \theta_{1} ; \quad \sum_{r} q_{2 r} A_{r}=d \theta_{2}
$$

recovering the canonical result (4.18) in a more general context. This reasoning answers the question posed earlier about why for linearly equivalent $D$ and $D^{\prime}, \mathcal{O}(D)$ and $\mathcal{O}\left(D^{\prime}\right)$ give the same Wilson line.

Before moving on, we study the vanishing of the generating set $A_{r}$ because of a possible relation to massless open strings. We wish to show that the $A_{r}$ will vanish at corners of $\Delta$ where $A_{r}$ is well defined. For this demonstration, we rely on a result of Abreu [47] that

$$
\operatorname{det}\left(g_{i j}\right)=\left[\delta(x) \prod_{r=1}^{n} \ell_{r}(x)\right]^{-1},
$$

where $\delta$ is a smooth function on $\Delta$. Since we are on a surface, at a corner of $\Delta$, the determinant of $g^{i j}$ involves a double zero, and it is straightforward to show that $g^{i j}$ must vanish. Since $g^{i j}$ vanishes, from (4.16) we see that $A_{r}$ will vanish as well unless the corner is associated with the vanishing of $\ell_{r}$.

\subsection{Bundles on $\mathbb{P}^{2}$}

To illustrate these ideas concretely, we present them for $\mathbb{P}^{2}$. There are three Weil divisors $D_{1}, D_{2}$, and $D_{3}$ on $\mathbb{P}^{2}$ corresponding to the three rays of the fan $v_{1}=(1,0), v_{2}=(0,1)$, and $v_{3}=(-1,-1)$. From (4.17), the vector potentials for the corresponding three line bundles, which in this case satisfy the equations of motion, are

$$
\begin{aligned}
& A_{1}=-\frac{1}{3}\left(x_{1}-2\right) d \theta_{1}-\frac{1}{3}\left(1+x_{2}\right) d \theta_{2}, \\
& A_{2}=-\frac{1}{3}\left(1+x_{1}\right) d \theta_{1}-\frac{1}{3}\left(x_{2}-2\right) d \theta_{2}, \\
& A_{3}=-\frac{1}{3}\left(1+x_{1}\right) d \theta_{1}-\frac{1}{3}\left(1+x_{2}\right) d \theta_{2},
\end{aligned}
$$

where the $x_{i}$ lie inside the triangle defined by $x_{1}>-1, x_{2}>-1$ and $x_{1}+x_{2}<1$. These $A_{r}$ are all gauge equivalent to each other, which is expected since the corresponding divisors are all linearly equivalent. The gauge transformation takes the form $A \rightarrow A+d \lambda$ where $\lambda=n_{1} \theta_{1}+n_{2} \theta_{2}$ and $n_{i}$ is an integer. The Wilson line corresponding to the $A_{r}$ will not change because the gauge transformation respects the periodicity of this square torus of height and length one. Thus we see that $\mathcal{O}\left(D_{1}\right), \mathcal{O}\left(D_{2}\right)$ and $\mathcal{O}\left(D_{3}\right)$ appear as the same point on $\tilde{T}^{2}$. Indeed, for any line bundle of the form $\mathcal{O}\left(a D_{1}+b D_{2}+c D_{3}\right)$, the point on the torus will depend only on $a+b+c$. Any line bundle of the form $\mathcal{O}\left(a D_{1}+b D_{2}+c D_{3}\right)$ can equivalently be written as $\mathcal{O}(a+b+c)$. 
We can take the vector potential corresponding to $\mathcal{O}(n)$ to be

$$
-\frac{n}{3}\left(1+x_{1}\right) d \theta_{1}-\frac{n}{3}\left(1+x_{2}\right) d \theta_{2}
$$

Thus, given the exceptional collection $\mathcal{O}, \mathcal{O}(1), \mathcal{O}(2)$, we should plot points at $p_{1}=(0,0), p_{2}=$ $\left(-1-x_{1},-1-x_{2}\right) / 3$ and $p_{3}=2\left(-1-x_{1},-1-x_{2}\right) / 3$ or their translates on $\tilde{T}^{2}$. These three points correspond to the $\mathrm{D}$-branes.

To connect these three $\mathrm{D}$-branes with open strings, we return to the $A_{i}$ (4.24)-4.26). Between $\mathcal{O}$ and $\mathcal{O}(1)$ or between $\mathcal{O}(1)$ and $\mathcal{O}(2)$, there are three possible paths corresponding to $D_{1}, D_{2}$, and $D_{3}$. The path corresponding to $D_{i}$ is defined by the Wilson line associated to $A_{i}$. Instead of thinking of the Wilson line as a point on the torus, we now think of it as a vector that joins two points. The resulting Beilinson quiver for $\mathbb{P}^{2}$ is shown in Figure 13. We do not need to draw in additional arrows corresponding to maps between $\mathcal{O}$ and $\mathcal{O}(2)$. All the requisite maps can be formed by joining together the arrows already drawn.

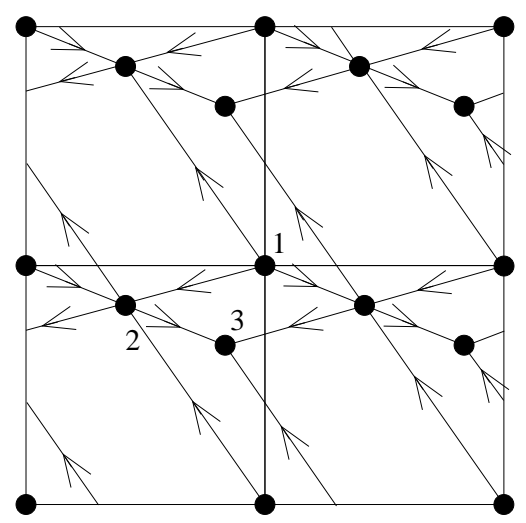

Figure 13: Four unit cells of the $\mathbb{P}^{2}$ periodic quiver for basepoint $\left(x_{1}, x_{2}\right)=(3 / 4,-1 / 2)$.

These vectors corresponding to the $D_{i}$ shrink to zero size at special base points on the polytope $\Delta$. In particular, the string corresponding to $D_{1}$ shrinks to zero at $(2,-1), D_{2}$ shrinks to zero at $(-1,2)$, and $D_{3}$ shrinks to zero at $(-1,-1)$.

One startling feature of this Beilinson quiver is that the arrows will never cross, no matter what our choice of basepoint $\left(x_{1}, x_{2}\right)$. As the $\left(x_{1}, x_{2}\right)$ moves to the boundaries of $\Delta$, arrows may become parallel and the three points may touch, but the arrows never cross.

\subsection{Constructing the Quiver in General}

Given a set of generating field strengths for the $\mathcal{O}\left(D_{r}\right)$, we can construct a family of periodic quivers from an exceptional collection. A particular quiver in the family will depend on the choice of basepoint $\left(x_{1}, x_{2}\right) \in \Delta$. If the metric is of physical interest, e.g. it lifts to a Ricci flat metric on the cone and provides a starting point for AdS/CFT constructions, and the field strengths satisfy the equations of motion, we expect this periodic quiver to be the quiver of physical interest. Thus, 
the quiver we described for $\mathbb{P}^{2}$ should be the "correct" quiver. Unfortunately, we in general do not have explicit expressions for the metric and the field strengths, only the canonical representatives detailed above.

In the absence of physical data, we will work with the canonical metric and hope that the resulting quiver is topologically if not geometrically accurate. Because we only expect topological data, we will fix a particularly convenient choice of basepoint in $\Delta:\left(x_{1}, x_{2}\right)=(0,0)$. In this case, the vector potential becomes

$$
A_{r}=\frac{1}{2} g^{k l} v_{r, l} d \theta_{k} .
$$

From this vector potential, we see that a general line bundle of the form $\mathcal{O}\left(\sum_{r} a_{r} D_{r}\right)$ will be plotted on the torus with coordinates

$$
\left(\sum_{r} q_{1 r} a_{r}, \sum_{r} q_{2 r} a_{r}\right)
$$

where

$$
q_{i r}=\frac{1}{2} g^{i l} v_{r, l}
$$

These two $q_{i r}$ are orthogonal to the $Q_{a}$ and are in fact the same as the $q_{i}$ discussed previously. Because $g^{k l}$ is complicated and we are after only topological information, let us rescale the $q_{i r}$ and the associated torus by a $g_{k l} \in G L_{2}(\mathbb{R})$ transformation, choosing $q_{j r}=v_{r, j}$ as before.

The procedure for constructing the quiver is very simple. Given a strongly exceptional collection of line bundles $\mathcal{E}=\left(E_{1}, E_{2}, \ldots, E_{n}\right)$, take $E_{j}=\mathcal{O}\left(\sum_{r} a_{r} D_{r}\right)$ and $E_{k}=\mathcal{O}\left(\sum_{r} b_{r} D_{r}\right)$. The homomorphisms from $E_{j}$ to $E_{k}$ are generated by the global sections of $\mathcal{O}\left(\sum_{r}\left(b_{r}-a_{r}\right) D_{r}\right)$. Start with the monomial

$$
\prod_{r} X_{r}^{b_{r}-a_{r}}
$$

This monomial has charges $\sum_{r} Q_{a r}\left(b_{r}-a_{r}\right)$. To be a global section, $b_{r}-a_{r} \geq 0$ for all $r$ (or there will be a pole). However, there may be more than one such monomial with this charge. Construct all such monomials. Call the set of such monomials $M_{j k}$. For each $m \in M_{j k}$, where $m=\prod_{r} X_{r}^{c_{r}}$, we compute

$$
\left(\phi_{1}, \phi_{2}\right)=\left(\sum_{i} q_{1 r} c_{r}, \sum_{i} q_{2 r} c_{r}\right)
$$

This vector $\left(\phi_{1}, \phi_{2}\right)$ is the relative position of nodes $j$ and $k$ on $\tilde{T}^{2}$. Fixing the position of $E_{1}$, we now have specified the location of all the nodes of the quiver.

Instead of a $\tilde{T}^{2}$ of length and height one as before, because of the rescaling, the period vectors of this torus are the $q_{i}$. If we take two points of the quiver separated by $a q_{1}+b q_{2}$, in the language of line bundles, we have $\mathcal{O}(D)$ and $\mathcal{O}\left(D+\sum_{r}\left(a q_{1 r}+b q_{2 r}\right) D_{r}\right)$. However, since the $q_{i}$ are orthogonal to the $Q_{i}, D$ and $D+\sum_{r}\left(a q_{1 r}+b q_{2 r}\right) D_{r}$ have the same $Q$ charges and are linearly equivalent as divisors. In other words, these two points are the same. 
Starting with the set $M_{k, k+1}$, we draw an arrow from node $k$ to node $k+1$ for each $m \in$ $M_{k, k+1}$. We repeat this procedure for line bundles of the form $E_{k}$ and $E_{k+2}$. There is an additional complication now. It may happen that the monomial $m=m_{1} m_{2}$ where $m_{1}$ joins nodes $E_{k}$ with $E_{k+1}$ and $m_{2}$ joins nodes $E_{k+1}$ and $E_{k+2}$. If such is the case, then we do not add an arrow corresponding to $m$. The entries of $\chi\left(E_{i}^{\vee}, E_{j}^{\vee}\right)$ let us know how many arrows we should be writing down. Recursively, we consider $E_{k}$ and $E_{k+i}$ and continue until all the arrows in the Beilinson quiver are drawn.

Take $\mathbf{d} \mathbf{P}_{1}$ to illustrate these ideas. $\mathrm{A}$ fan is $v_{1}=(0,1), v_{2}=(1,1), v_{3}=(0,-1)$, and $v_{4}=(-1,0)$ from which we choose

$$
q=\left(\begin{array}{cccc}
0 & -1 & 0 & 1 \\
-1 & -1 & 1 & 0
\end{array}\right) .
$$

An exceptional collection on $\mathbf{d P}_{\mathbf{1}}$ is $\mathcal{O}, \mathcal{O}\left(D_{1}\right), \mathcal{O}\left(D_{4}+D_{1}\right), \mathcal{O}\left(D_{4}+D_{1}+D_{3}\right)$. Using the procedure described above, we find the Beilinson quiver, figure 14 .

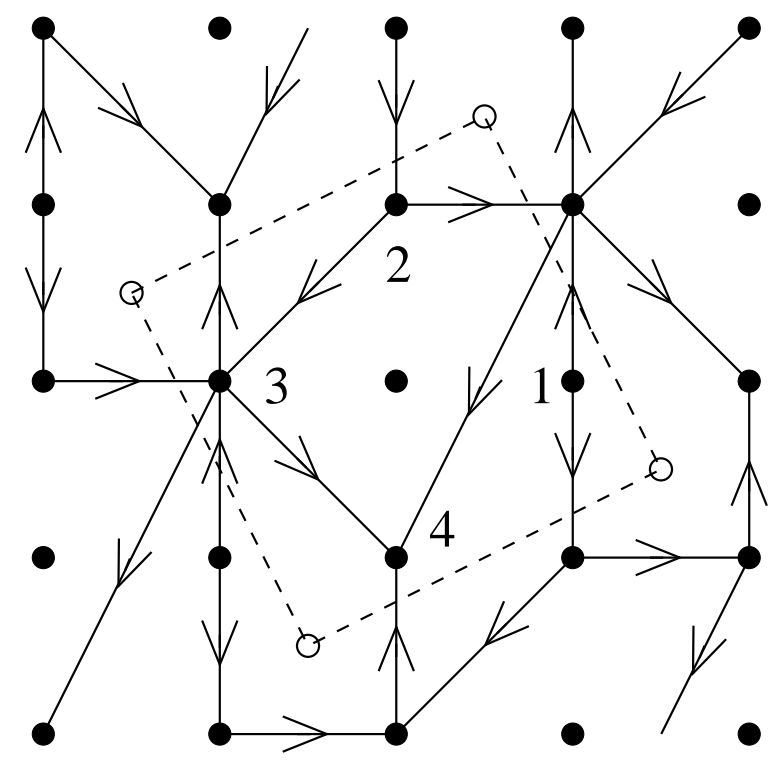

Figure 14: The periodic Beilinson quiver for $\mathbf{d P}_{\mathbf{1}}$ with fundamental cell.

For example, consider the paths between $\mathcal{O}\left(D_{4}+D_{1}\right)$ and $\mathcal{O}\left(D_{4}+D_{1}+D_{3}\right)$. We look for all monomials with the $Q$ charges of $D_{3}$, in other words $x_{3}, x_{1} x_{4}$, and $x_{1} x_{2}$. These three monomials have torus charges $q,(0,1),(1,-1)$, and $(-1,-2)$ respectively. On our torus, node 4 is indeed at relative positions $(0,1),(1,-1)$, and $(-1,-2)$ to node 3 with corresponding arrows drawn in.

\subsection{Vanishing Euler Character}

We can argue that the Euler character of the torus (to be distinguished from the Euler character of the exceptional collection) must vanish and so the most obvious obstruction to writing the quiver on 
a torus is eliminated. (Of course, we don't have an arbitrary collection of lines, vertices, and faces, but have instead completely specified the connectivity, and it remains unclear that the pattern of connectivity will be compatible with a torus structure.) Given exceptional collections $\mathcal{E}$ and $\mathcal{E}^{\vee}$, in terms of charges, we can decompose any sheaf $F$ into the $E_{j}^{\vee}$ or the $E_{j}$ :

$$
\operatorname{ch}(F)=\sum_{j} \chi\left(E_{j}, F\right) \operatorname{ch}\left(E_{j}^{\vee}\right) ; \quad \operatorname{ch}(F)=\sum_{j} \chi\left(F, E_{j}^{\vee}\right) \operatorname{ch}\left(E_{j}\right) .
$$

We are interested in quivers that come from a stack of D3-branes, which look like a point in $V$. Thus, for a skyscraper sheaf

$$
\operatorname{ch}\left(\mathcal{O}_{p t}\right)=\sum_{j} \chi\left(E_{j}, \mathcal{O}_{p t}\right) \operatorname{ch}\left(E_{j}^{\vee}\right)=\sum_{i, j} \chi\left(E_{j}, \mathcal{O}_{p t}\right) \chi\left(E_{j}^{\vee}, E_{i}^{\vee}\right) \operatorname{ch}\left(E_{i}\right)
$$

The rank component of the chern class of a skyscraper sheaf vanishes, and $\chi\left(E_{i}, \mathcal{O}_{p t}\right)=\operatorname{rk}\left(E_{i}\right)$. Thus,

$$
0=\sum_{i, j} \operatorname{rk}\left(E_{i}\right) \operatorname{rk}\left(E_{j}\right) \chi\left(E_{i}^{\vee}, E_{j}^{\vee}\right)
$$

For these toric exceptional collections, we find exceptional collections of line bundles where the ranks are all one. Thus, the sum over the entries of the Euler character must vanish. But this sum has a different interpretation. The sum over the diagonal entries is the number of gauge groups. The sum over the negative entries is the number of arrows in the Beilinson quiver, and the sum over the off-diagonal positive entries is the number of relations:

$$
\sum_{i, j} \chi\left(E_{i}^{\vee}, E_{j}^{\vee}\right)=\text { gauge groups }- \text { arrows }+ \text { relations } .
$$

Now for these toric quivers, we know that each relation corresponds to two superpotential terms. Moreover, when we lift to the Calabi-Yau quiver, each relation also becomes an additional arrow. Thus, for the Calabi-Yau quiver

$$
\text { gauge groups }- \text { arrows }+ \text { superpotential terms }=0
$$

which is exactly the condition that the Euler character of the torus vanish because for each gauge group we have a node, for each arrow an edge, and each superpotential term a face in the quiver. ${ }^{9}$ Moving back to the Beilinson quiver now consists of removing a set of arrows, which cannot change the Euler character of the graph. This demonstration of vanishing Euler character is complementary to but distinct from a similar observation in [2] where the authors use $\mathrm{R}$-charge constraints to prove that the Euler character of the brane tiling vanishes.

\footnotetext{
${ }^{9}$ We would like to thank Aaron Bergman for this observation relating $\chi\left(E_{i}^{\vee}, E_{j}^{\vee}\right)$ to the Euler character of the torus.
} 


\section{Compatibility}

Having established that one can derive periodic quivers from exceptional collections, we now study the possibility of generating such collections by means of brane tilings. In this section we define a map that assigns line bundles to paths in the quiver. This map can be used to compute an exceptional collection on a complex surface that shrinks to zero size at the singularity. The exceptionality can be checked on a case-by-case basis. Given these bundles, one can reconstruct the quiver based on mathematically rigorous procedures [4, 0, 8, 9, 10, 11, 12, 48]. By reinterpreting paths and perfect matchings in the tiling language, we explicitly prove that this construction gives back our original quiver.

\subsection{Beilinson quivers and internal matchings}

For the exceptional collection technique to be useful when applied to toric Calabi-Yau manifolds, we need the toric diagram to contain at least one internal point. This restriction means that our manifold can be partially resolved by blowing up a 4-cycle. Let us consider the tiling for this Calabi-Yau which can be most efficiently constructed by the Fast Inverse Algorithm [15, 16]. Let us also fix a reference internal matching $P M_{0}$ that resides at one of the internal points of the toric diagram. We can set the origin at this point.

If we remove those bifundamentals from the quiver that are contained in $P M_{0}$, then we obtain another smaller quiver. We will show that this subquiver contains no oriented loops and therefore has the right properties to be a Beilinson quiver for the relevant $4-$ cycle. $^{10}$ For an example see Figure 2. This Beilinson quiver is generated by deleting the bifundamentals that are contained in the $4^{\text {th }}$ perfect matching of Figure 9. Recall that the Beilinson quiver was defined at the beginning of Section 1 from an exceptional collection. Here, we define an intermediate notion

Definition 5.1.1. We define a pre-Beilinson quiver to be a connected subquiver of the gauge theory quiver that contains no oriented loops and all the nodes of the original.

Let us summarize some additional terminology we use in the following.

Definition 5.1.2. An oriented path is a path in the quiver that respects the direction of the arrows.

Definition 5.1.3. Paths in the quiver that also exist in a Beilinson (or pre-Beilinson) quiver are called allowed paths.

We say that a path crosses an edge in the tiling if the path contains the corresponding arrow in the quiver. Paths that exist in the Beilinson quiver will not intersect the edges of $P M_{0}$. It is easy to see that $\mathrm{F}$-terms transform allowed paths to allowed paths. Closed paths may wind

\footnotetext{
${ }^{10}$ We would like to thank Robert Karp for discussion about this point.
} 
around the tiling torus, and the winding can be characterized by the homology class of the loop $(p, q)$. The $(0,0)$ loops are called trivial loops. By definition, the length of an oriented path is the $\mathrm{R}$-charge of the corresponding operator. Paths can be related by $\mathrm{F}$-term transformations, but these transformations will not change the total $\mathrm{R}$-charge associated to a path. The height functions of the external matchings with respect to $P M_{0}$ are called height coordinates.

Lemma 5.1.4. In a consistent tiling, an internal perfect matching determines a pre-Beilinson quiver by removing those bifundamentals from the quiver that are contained in the matching.

Proof. Removing bifundamentals from the gauge theory quiver that are contained in $P M_{0}$ does not remove nodes and does not create disconnected pieces. The nontrivial part of the proof involves the oriented loops.

(i) First we show that trivial allowed loops cannot exist. Such a loop would contain at least one edge $e$. By crossing this edge in the tiling, some of the height functions would increase by one. The increase happens exactly when the corresponding perfect matchings contain $e$. Allowed paths will never go "downhill" on the graph of any height function, because then they would have to cross an edge in $P M_{0}$ which is not allowed (the edge is not present in the pre-Beilinson quiver). See Figure 15 for the schematic picture. The increase of the height function is "irreversible", i.e. the function is monotone along an allowed path; hence we have arrived at a contradiction.

For this argument to hold one has to show that $e$ is contained in at least one perfect matching. We can suppose this, since otherwise we can omit this edge from the tiling and still get the same toric diagram which questions the consistency of the original tiling.

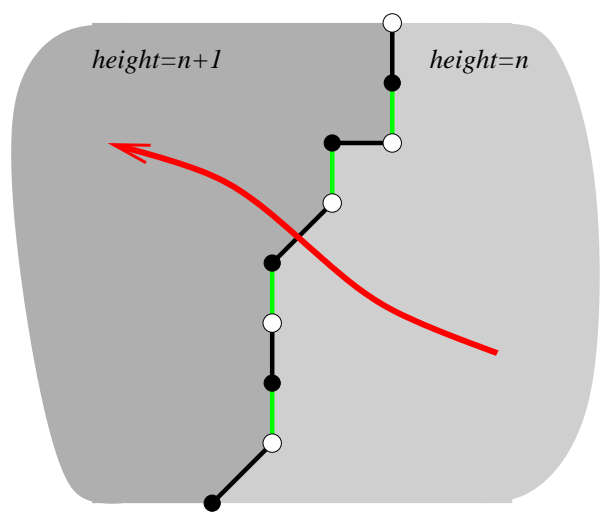

Figure 15: Allowed face paths (i.e. paths in the Beilinson quiver) go always uphill. The height function increases by one at the line constituted of the black perfect matching and the green reference matching. The red path cannot cross the green edges (they are not in the Beilinson quiver). Hence when crossing the contour line, the red path has to cross a black edge. Crossing the black edge increases the value of the height function.

(ii) We also need to show that there are no non-trivial loops in the pre-Beilinson quiver. These 
non-trivial loops wrap the torus cycles. Suppose that there exists such a loop. This oriented loop is a face path on the brane tiling with homology class $(x, y) \in \mathbb{Z}^{2}$ as in Figure 16. Let us take an arbitrary external matching $P M_{i}$ at $\left(s_{i}, t_{i}\right)$. We can compute the height function assigned to this matching with respect to $P M_{0}$.

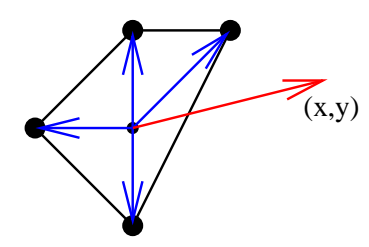

Figure 16: Gradient vectors in the toric diagram. The coordinates of the blue $\left(s_{i}, t_{i}\right)$ vectors give the monodromy of the height function of the perfect matching sitting at their endpoints. The red $(x, y)$ arrow is the gradient vector of the hypothetical nontrivial loop.

The height function should not decrease along the path. As an immediate consequence, the scalar product $\left(s_{i}, t_{i}\right) \cdot(x, y)$ must be nonnegative. On the other hand, the set of vectors $\left\{\left(s_{i}, t_{i}\right)\right\}$ span the whole $2 \mathrm{~d}$ space with positive coefficients, and thus at least one of these vectors has negative scalar product with $(x, y)$. This is a contradiction; therefore the pre-Beilinson quiver doesn't contain non-trivial loops.

\subsection{Line bundles from tiling: The $\Psi-$ map}

In the last section we saw that a candidate Beilinson quiver could be created from an internal perfect matching. In this section we continue by defining a map $\Psi$ that assigns a divisor to an allowed path by using external perfect matchings. We conjecture that these divisors give exceptional collections of line bundles which we will use to reconstruct the Beilinson quiver.

A Weil divisor can be represented by an integer function over the external vertices of the toric diagram polygon (see Figure 17). We call two such integer functions equivalent if they differ by a linear function $f(x, y)=x m+y n$ which defines a principal divisor. (Here $x$ and $y$ are coordinates on the plane of the polygon.)

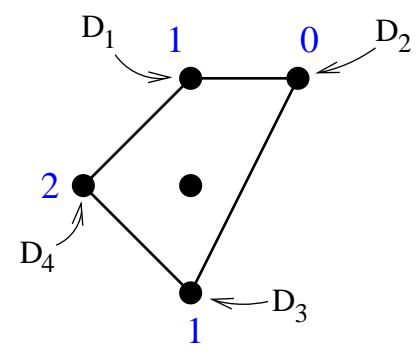

Figure 17: An integer function over the external nodes determines a divisor and therefore a sheaf of sections of the corresponding line bundle. The numbers in the figure denote $\mathcal{O}\left(D_{1}+D_{3}+2 D_{4}\right)$. 
Let us fix an arbitrary oriented path $P$. Then, $\Psi(P)$ gives a divisor, i.e. an integer function over the external nodes. We define this map by using the matchings of the tiling. For each external node $v_{r}$, there is a corresponding unique perfect matching ${ }^{11} P M_{r}$. We assign to the divisor $D_{r}$ the integer $\Psi_{r}(P)$ that is the number of edges in $P M_{r}$ which are crossed by the path $P$. In Figure 18 we see an example.

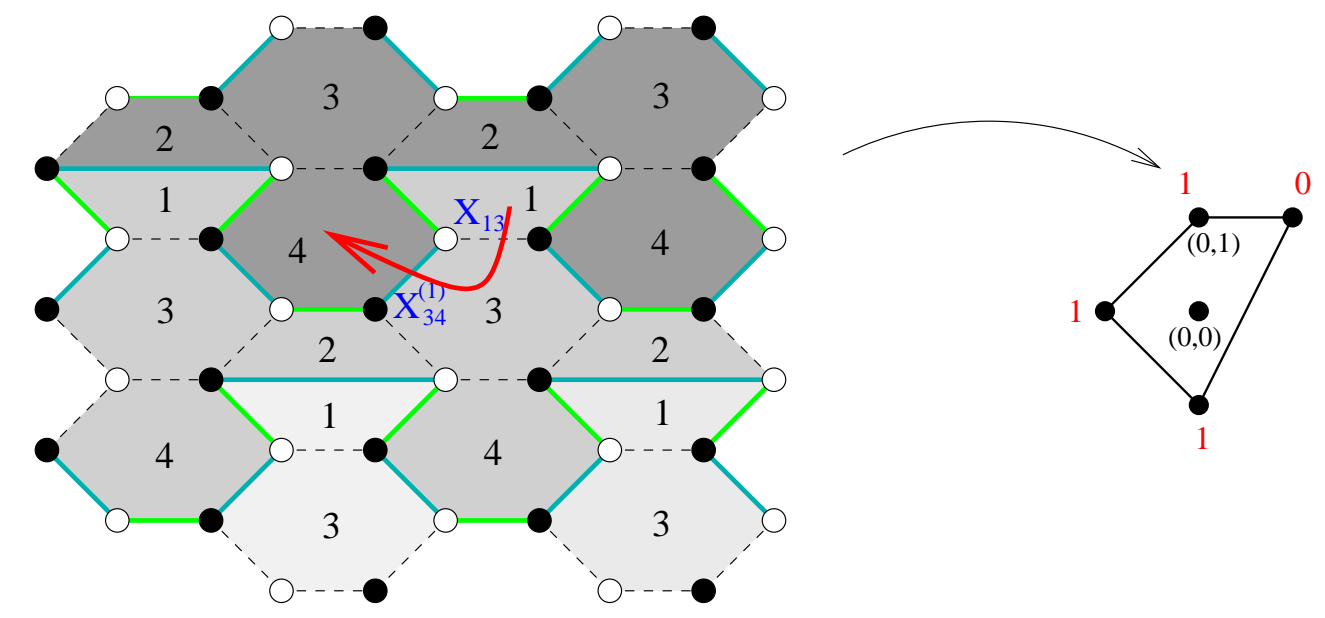

Figure 18: The $\Psi$-map.

The left hand side shows the brane tiling for $\mathbf{d P}_{1}$. The red path $P$ crosses two edges; hence it labels the operator $X_{13} \cdot X_{34}^{(1)}$. There is a corresponding oriented $1 \rightarrow 3 \rightarrow 4$ path in the quiver as in Figure 1. We have chosen the 4th matching from Figure 9 as the green reference matching. To show how to compute $\Psi_{8}(P)$, we have drawn the 8th matching of Figure 9 (in blue). The shading of the faces indicates the height function of this matching that has $(0,1)$ monodromy. The red path crosses one blue edge in the matching (namely $X_{34}^{(1)}$ ); hence $\Psi_{8}(P)=1$. One can compute the other integer "intersection numbers" with the help of the other external perfect matchings. The resulting numbers are indicated in red. These numbers define a Weil divisor on the base of the threefold. The numbers can also be interpreted as the increase in the height coordinates as we go along the path $P$. If the path is an allowed path (Definition 5.1.3) starting at face $A$ and ending at $B$, then $\Psi_{r}$ is simply the $h_{r}(B)-h_{r}(A)$ difference in the height function that corresponds to the $r^{\text {th }}$ external node. $\Psi$ is a well-defined function on the paths of the quiver. In fact, it does not depend on the choice of the reference perfect matching (modulo linear equivalence).

The $\Psi$-map can be extended to unoriented paths, i.e. paths that do not respect the arrow direction in the quiver. When crossing an edge in $P M_{i}$ in the reverse direction, we subtract one instead of adding one in computing $\Psi_{r}(P)$.

Let $C_{i}$ denote the Abelian group of chains in the periodic quiver. Here the quiver is understood

\footnotetext{
${ }^{11}$ We assume that the tiling is consistent and there are no "external multiplicities", i.e. there is a unique perfect matching corresponding to each external node of the toric diagram.
} 
as a discretization of the 2 -torus. This is the free group generated by the edges in the quiver with integer coefficients. The elements of $C_{1}$ take the following form

$$
P=\sum_{i} c_{i} X_{i} \quad\left(c_{i} \in \mathbb{Z}\right)
$$

where $X_{i}$ denotes the $i^{\text {th }}$ edge. We denote the cycles in $C_{i}$ by $Z_{i}$ and the boundaries by $B_{i}$. Elements of $B_{1}$ are built out of trivial loops. $\Psi$ can be extended in a straightforward way to be defined on $C_{1}$

$$
\Psi_{r}=\sum_{j} c_{p_{j}}
$$

where $\left\{p_{j}\right\}$ is the list of edges in the $r^{\text {th }}$ external matching. In the following, we will study the properties of this extended $\Psi$-map.

For an elementary loop around a node in the tiling, the image of $\Psi$ is a constant function (the anticanonical class $K$ ). Since all the perfect matchings cover this node, each matching is intersected by the loop precisely once; hence $\Psi_{r}=1$ for all $r$. This coincides with the observations made in [3]. In fact, one can easily prove that the entire $B_{1}$ subgroup is mapped to constant functions.

Gauge invariant mesonic operators can be constructed from arbitrary oriented loops ${ }^{12}$. These are the elements of $Z_{1}$. For these loops $\Psi$ assigns non-negative affine functions on the toric diagram parametrized by three integers. These functions are points in the dual cone. This is being investigated in 51].

We will now use $\Psi$ to compute a collection of line bundles. We choose an internal reference matching which determines a Beilinson quiver and therefore an ordering of the faces in the tiling. Without losing generality, we relabel the groups such that there are no arrows from node $i$ to $j$ if $i>j$.

Let us fix an allowed path $P_{i}$ for each face in the tiling (for $\mathbf{d} \mathbf{P}_{1}$ see Figure 19). We will call $\left\{P_{i}\right\}$ the set of reference paths. We choose these paths such that they start on face 1 and end on the specific face. This is possible because the Beilinson quiver is connected. Then, $\Psi$ maps each of these paths to a Weil divisor (see Figure 20 for the image). These divisors determine a collection of line bundles.

There is a general freedom in the choice of these paths. The terminal faces can also be chosen from different fundamental cells. We demonstrate this ambiguity in Figure 21. Let us pick two different paths that end on the same faces but in different fundamental cells. Recall that $\Psi$ maps closed loops to linear functions; hence the difference of the resulting divisors is linear, which means that they are in fact equivalent. Note that $\Psi$ gives the same set of integers for operators (paths) related by $\mathrm{F}$-term equations.

\footnotetext{
${ }^{12}$ Related work on mesonic operators was recently done in 49 , 50].
} 


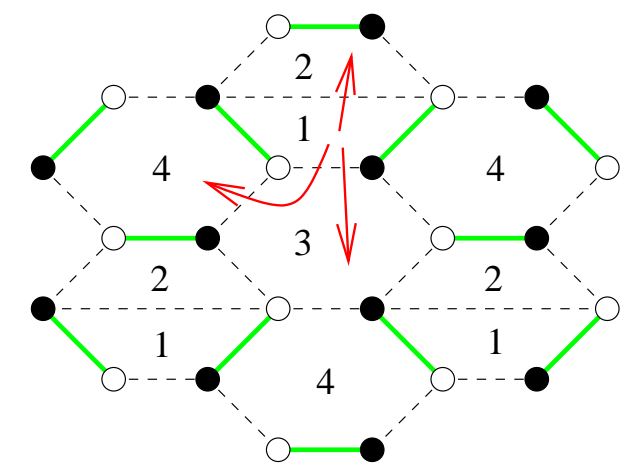

Figure 19: The reference paths are allowed paths to each face. They start from face 1 and don't cross the edges of the green internal matching; hence they are paths in the Beilinson quiver.

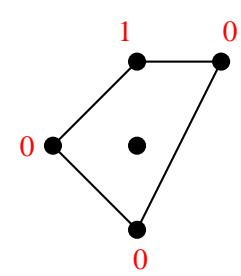

$1 \rightarrow 2$

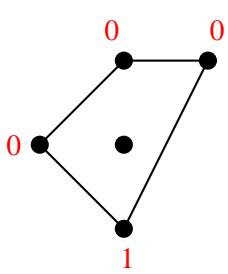

$1 \rightarrow 3$

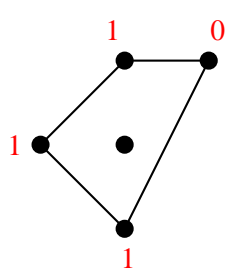

$1 \rightarrow 3 \rightarrow 4$

Figure 20: The three divisors computed from the paths to the faces.
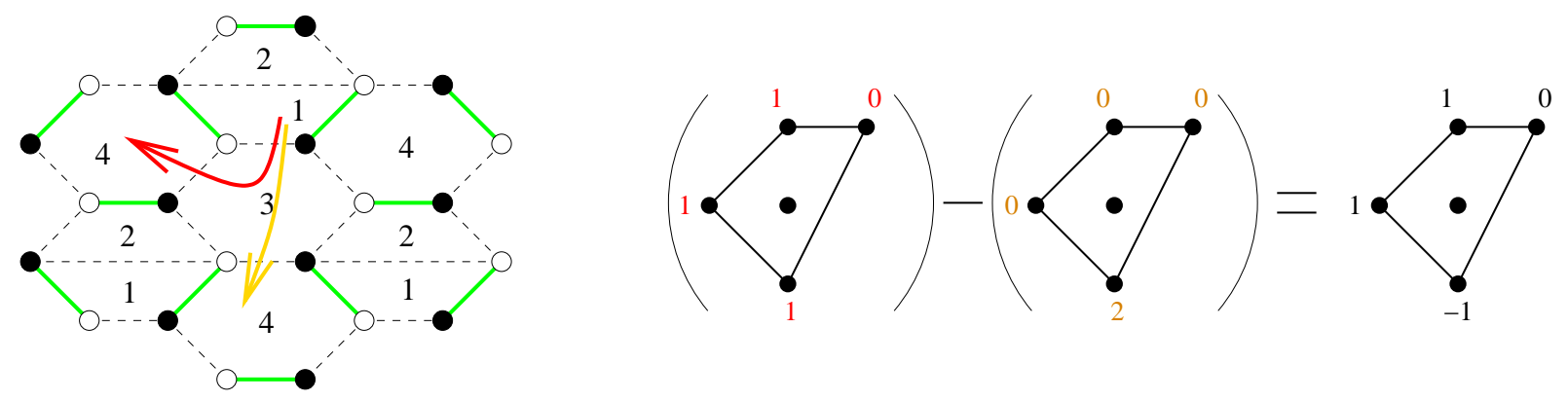

Figure 21: Face 4 can be assigned with either the red or the yellow allowed path. The resulting Weil divisors are shown on the right-hand side. We see that they differ by a linear function, i.e. they are equivalent.

After determining the divisors that correspond to the $P_{i}$ paths, we are ready to write down an exceptional collection. We introduce the notation

$$
\mathcal{O}\left(\sum_{r} a_{r} D_{r}\right) \equiv\left(a_{1}, a_{2}, \ldots, a_{n}\right)
$$

We assign the line bundle of the divisor $\Psi\left(P_{i}\right)$ to the $i^{\text {th }}$ face. The integer numbers sitting at the external nodes are the $a_{i}$ coefficients. For the first face we assign $(0,0, \ldots, 0)$. In our $\mathbf{d P}_{1}$ example 
from Figure 20 we obtain the following collection:

$$
(0,0,0,0),(1,0,0,0),(0,0,1,0),(1,0,1,1)
$$

which is exactly the collection discussed in section 4.4.

Another example for the $Y^{3,2}$ theory is presented in the Appendix.

Before moving on, we would like to point out that the $\Psi$-map efficiently computes the divisors that correspond to dibaryons. In order to obtain the divisor for the bifundamental $X$, we simply compute $\Psi(X)$. For $\mathbf{d P}_{1}$ we get the following list

\begin{tabular}{|c|c|}
\hline field & divisor \\
\hline \hline$X_{12}$ & $(1,0,0,0)$ \\
$X_{23}^{(1)}, X_{23}^{(2)}$ & $(0,1,0,0) \cong(0,0,0,1)$ \\
$X_{41}^{(1)}, X_{41}^{(2)}$ & $(0,1,0,0) \cong(0,0,0,1)$ \\
$X_{42}$ & $(0,0,1,0)$ \\
$X_{13}$ & $(0,0,1,0)$ \\
$X_{34}^{(1)}, X_{34}^{(2)}, X_{34}^{(3)}$ & $(0,0,1,0) \cong(1,1,0,0) \cong(1,0,0,1)$ \\
\hline
\end{tabular}

in precise agreement with section 5.1 of [14]. The linear equivalence relations $\cong$ are easily established. Let us show that $(0,0,1,0) \cong(1,0,0,1)$. The difference divisor $(1,0,0,1)-(0,0,1,0)=$ $(1,0,-1,1)$, shown on the right hand side of Figure 21, has a $\Psi$ map of the form $\Psi=y-x$. In other words $(1,0,-1,1)$ is a principal divisor and the linear equivalence follows.

In this section we defined the linear $\Psi$-map that computes the divisors corresponding to the bifundamental fields. This map can be used explicitly to write down a collection of line bundles for the singularity. Unfortunately, we are lacking a general proof that the generated collections are always exceptional. Strong exceptionality may be checked on a case-by-case basis. 


\subsection{Reconstructing the quiver}

In section 5.2 we introduced the general method, the $\Psi$-map, that computes a collection of line bundles that is presumably strongly exceptional. Given such a collection, we can use rigorous methods to construct the quiver of the gauge theory. In this section we prove that the quiver obtained this way matches with the dual graph of the tiling which was our starting point. ${ }^{13}$

Let us denote the exceptional collection by $\left\{E_{i}\right\}$. We define the matrix

$$
S_{i j}=\operatorname{dim} \operatorname{Hom}\left(E_{i}, E_{j}\right) .
$$

The matrix elements in $S$ tell the number of ways of getting from node $i$ to node $j$ in the Beilinson quiver, taking the relations into account. The inverse of this matrix gives the quiver directly up to bidirectional arrows. The nonzero elements of $S_{i j}^{-1}(i<j)$ are the number of arrows from $j$ to $i$ minus the number of arrows from $i$ to $j$ in the quiver.

Since we are dealing with line bundles on toric manifolds, the computation of $\operatorname{dim} \operatorname{Hom}\left(E_{i}, E_{j}\right)$ gets vastly simplified [21]. This dimension is equal to the number of global sections of the bundle $E_{j} \otimes E_{i}^{*}$, which we denote by $\mathcal{O}\left(\sum_{r} a_{r} D_{r}\right)$. Then, the dimension is obtained by counting the lattice points inside the polygon

$$
\Delta_{i j}=\left\{u \in \mathbb{R}^{2}: u \cdot v_{r} \leq a_{r} \text { for all } r\right\}
$$

where $v_{r} \in \mathbb{Z}^{2}$ is the position of the $r$ th external node in the toric diagram. See the left-hand side of Figure 22 for an example.

In section 5.2 we computed the (5.4) exceptional collection for $\mathbf{d P}_{1}$. Using the above described method, the $S$ matrix and its inverse are determined

$$
S=\left(\begin{array}{llll}
1 & 1 & 3 & 6 \\
0 & 1 & 2 & 5 \\
0 & 0 & 1 & 3 \\
0 & 0 & 0 & 1
\end{array}\right) \quad S^{-1}=\left(\begin{array}{cccc}
1 & -1 & -1 & 2 \\
0 & 1 & -2 & 1 \\
0 & 0 & 1 & -3 \\
0 & 0 & 0 & 1
\end{array}\right)
$$

We see that $S^{-1}$ gives precisely the quiver in Figure 11.

In the following, we will show that this lattice point counting method of determining the number of paths from node $i$ to node $j$ in the quiver is identical to the same computation on the brane tiling. Since the number of paths essentially encodes the quiver via $S$ and $S^{-1}$, we are proving that the collection of line bundles encodes the quiver of the original brane tiling.

The key observation is that the lattice of $\Delta_{i j}$ can be identified with the lattice of fundamental cells of the brane tiling. ${ }^{14}$ This is shown in Figure 22. In particular, we will assign the lattice points to the $j^{\text {th }}$ faces in the cells. The simple counting of lattice points also counts the inequivalent

\footnotetext{
${ }^{13}$ We will prove this for the non-periodic McKay quiver.

${ }^{14}$ We thank Alastair King for related discussions.
} 


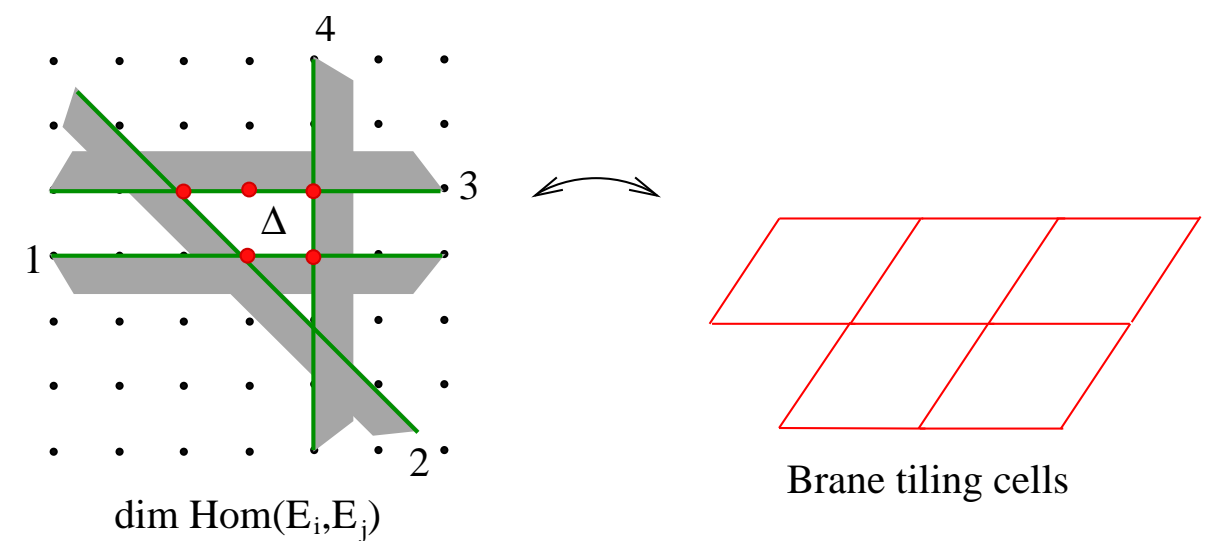

Figure 22: Determining the $S_{2,4}$ matrix element. In this case $E_{4} \otimes E_{2}^{*}=(1,0,1,1)-(1,0,0,0)=(0,0,1,1)$. The figure shows the lattice of the $\Delta_{2,4}$ polygon and its bounding inequalities. The red lattice points inside $\Delta_{2,4}$ can be identified with adjacent fundamental cells in the brane tiling.

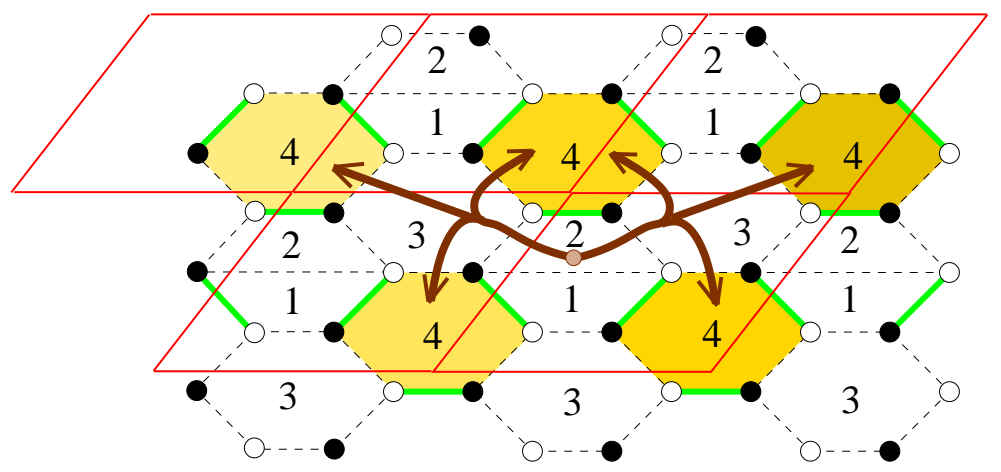

Figure 23: The figure shows the allowed paths that start on face 2 and end on face 4 . The endpoints of these paths are in different fundamental cells which are in one-to-one correspondence with the lattice points inside $\Delta_{2,4}$ that has been used to compute $\operatorname{dim} \operatorname{Hom}\left(E_{2}, E_{4}\right)$.

allowed paths from face $i$ to face $j$. There can be many such paths, but their number is finite, since no loops are allowed. The lattice points in $\Delta_{i j}$ are in one-to-one correspondence with adjacent fundamental cells that contain the final $j$ faces where these paths end. In Figure 23 these are the five faces marked in yellow. We see that to one of these faces there are two allowed paths leading. This shouldn't trouble us, since these are equivalent paths related by the $U_{2}^{1} V^{2}=U_{2}^{2} V^{1} \mathrm{~F}$-term equation for the $Y_{2}$ bifundamental field that separates face 2 and face 4 . In fact, it turns out that a general feature of consistent tilings is that homotopic paths of the same length (measured by the $\mathrm{R}$-charge of the corresponding trace operator ${ }^{15}$ ) are $\mathrm{F}$-term equivalent. In the following, we will prove this statement.

\footnotetext{
${ }^{15}$ In fact, any trial $\mathrm{R}$-charge can be used to measure the length.
} 
Lemma 5.3.1. In a consistent tiling, paths of the same length are F-term equivalent iff they are homotopic.

Proof. F-flatness equations are local transformations of the paths (Figure 24); hence they transform homotopic paths into one another. Applying such a transformation to the path does not change the $\mathrm{R}$-charge of the corresponding operator. We need to show that two homotopic paths are equivalent.

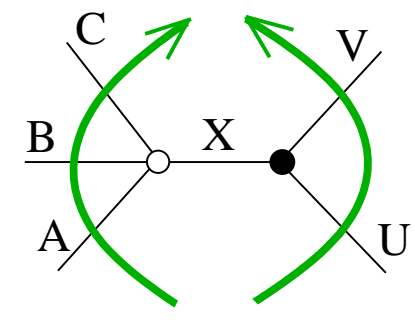

Figure 24: The $\mathrm{F}$-flatness equation for the $X$ bifundamental field is $C B A=V U$. This states the equivalence of the two green paths in the figure.

As an illustration, Figure 25 shows two such paths in a square lattice that can be deformed into one another by $\mathrm{F}$-terms. The rhombi they surround are also shown separately in the righthand side of the figure. This area has two bounding lines: $A A_{1} A_{2} A_{3} B$ and $A B_{1} B_{2} B_{3} B$. On the boundary we find two kinds of rhombus nodes alternating: Every other node is also a node of the tiling $\left(A_{1}, A_{3}, B_{1}, B_{3}\right)$. We call these odd nodes. The remaining even nodes $\left(A, A_{2}, B, B_{2}\right)$ are only vertices in the rhombus lattice.

We can start deforming path 1 by using the $\mathrm{F}$-term equation for the tiling edge $A_{3} B_{3}$. We also see that using the $\mathrm{F}$-term equation for $A_{1} B_{1}$ is not possible because path 1 does not contain $A_{1} B_{3}$. At the level of the rhombus lattice the difference of the two nodes $A_{3}$ and $A_{1}$ can be quickly seen: There is no red rhombus lattice edge in the pink area that connects $A_{3}$ to another node, whereas $A_{1}$ has one edge, namely $A_{1} B_{2}$. To summarize, the area between two paths can be reduced by F-terms where the boundary nodes don't have rhombus edges.

Let us consider two homotopic paths that start and end on the same two faces. For simplicity, we assume that the paths are not intersecting. We also assume that the area between the two paths has been completely reduced, i.e. there are no more F-terms that we can use to decrease it. This is equivalent to requiring that the odd nodes along the boundary have at least one rhombus edge going to the interior of the area. One can check that by construction the even nodes always have at least one rhombus edge. (In the previous example, such nodes were $A_{2}$ and $B_{2}$.) The reduced area can be schematically drawn as in Figure 26.

If we suppose that there is precisely one red rhombus edge at each $A_{i}$ and $B_{j}$ node and there are no edges at $A$ and $B$, then we recognize a straight rhombus path built out of the $r_{i}(i=0,1,2, \ldots, n)$ rhombi. These are located at the boundary next to path 1 (see Figure 27). 

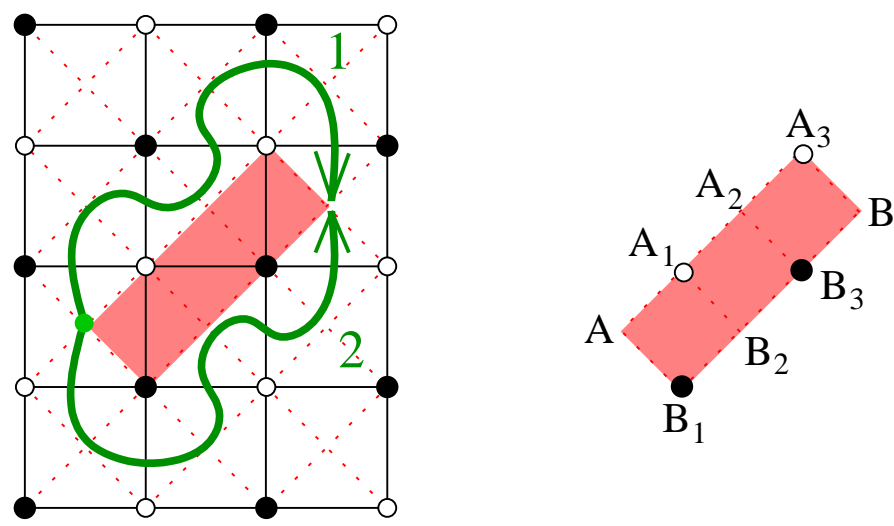

Figure 25: Homotopic paths are equivalent. The left-hand side of the figure shows two paths represented schematically by green lines. The tiling is colored black and the underlying rhombus lattice is shown by dotted lines. The pink area surrounded by the two paths is also shown separately.

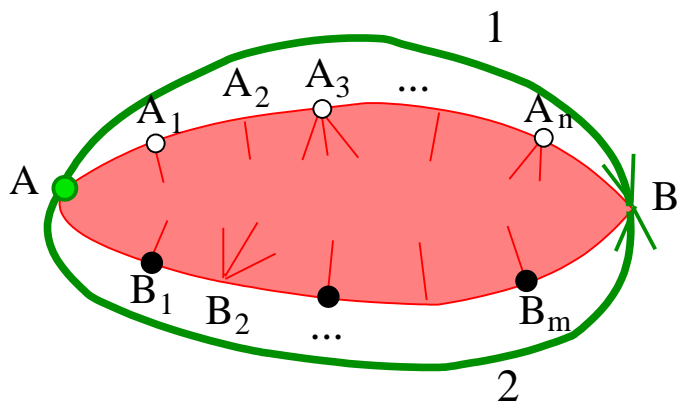

Figure 26: Two homotopic paths that pass around the pink area. Each boundary node $\left(A_{1}, \ldots, A_{n}, B_{1}, \ldots, B_{m}\right)$ has at least one rhombus edge which ensures that the area cannot be reduced by $\mathrm{F}$-terms.

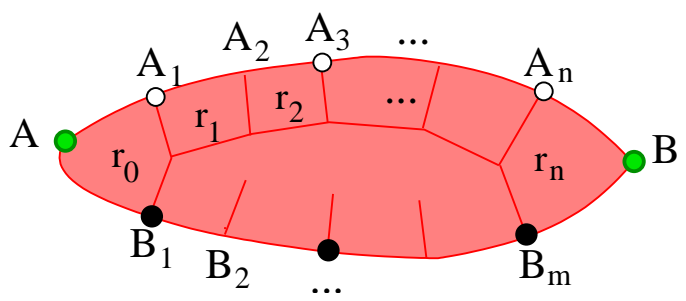

Figure 27: The straight rhombus path in the area contains rhombi $r_{0}, \ldots, r_{n}$. The existence of this series of rhombi constrains $A B_{1}$ to be parallel to $B_{m} B$.

This rhombus path corresponds to a zig-zag path in the tiling. The opposite edges of the rhombi are parallel; hence $A B_{1}$ is parallel to $B_{m} B$. The same argument applies for the rhombi on the other side of the area; hence $A A_{1}$ is parallel to $A_{n} B$. As a consequence, some of the rhombi in the area must be degenerate (here $r_{0}$ and $r_{n}$ ), i.e. the $\mathrm{R}$-charges of the corresponding fields are zero or negative and the tiling is inconsistent. Here we used that there is one rhombus edge for 
each node.

Extra rhombus edges joining to $A_{i}, B_{j}$ or to the endpoints $A$ or $B$ can't be used to restore the consistency of the tiling since they make the rhombi even more degenerate. This can also be seen by looking at the sum of internal angles of the $A, A_{1}, \ldots, A_{n}, B, B_{m}, \ldots, B_{1}, A$ pink polygon. This polygon has $n+m+2$ vertices, hence the sum of angles should be $(n+m) \pi$. Every rhombus next to the boundary contributes $\pi$ to the sum, except for the rhombi at $A$ and $B$ whose contribution can be bigger. If there are extra rhombus edges at a particular node, then we also get contribution from those rhombi that touch this node but they don't have a common edge with the boundary polygon. Since there are at least $n+m$ rhombi, the total sum of angles is greater than $(n+m) \pi$; hence the polygon must be degenerate.

As an immediate corollary, the lemma proves the following observation of [52]

Corollary 5.3.2. The structure of the chiral ring is naturally encoded in the non-trivial cycles of the tiling torus. In particular, the dual cone can be "embedded" in the infinite tiling [5].

The embedding is sketched in Figure 28.

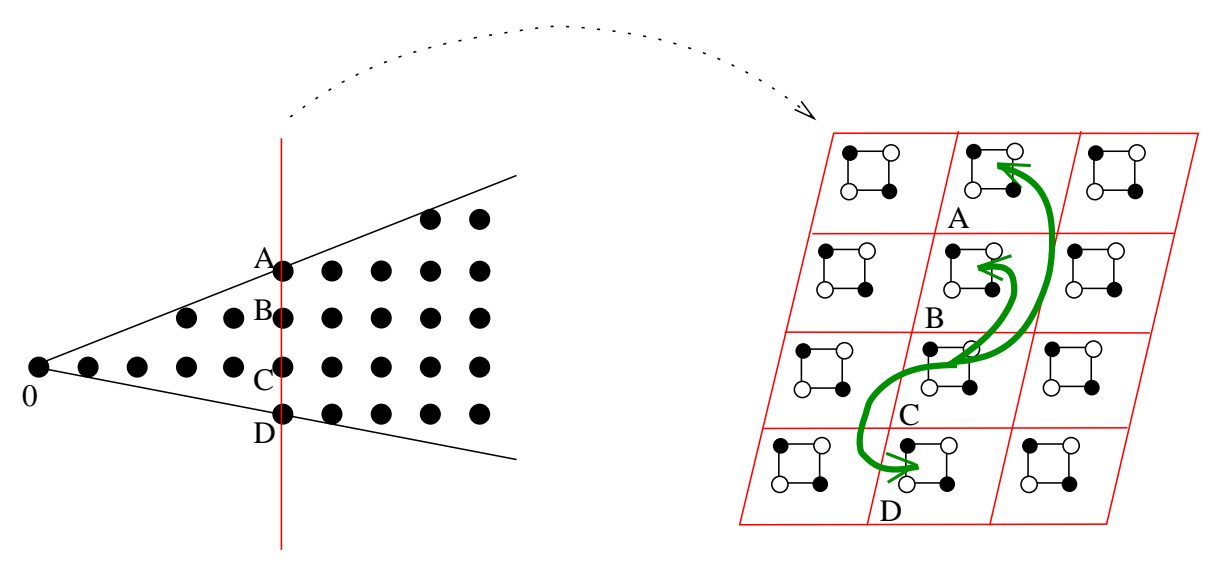

Figure 28: The embedding of the dual cone in the tiling torus.

One can assign gauge invariant mesonic operators to each of the monomials in the dual cone. For the $A, B, D$ monomials we assigned three green paths that are schematically shown in the right-hand side of the figure. They start and end on the same square in the tiling. Keeping these endpoints and the lengths fixed, they can be freely deformed due to Lemma 5.3.1.

Then, the endpoints of the paths in the lattice of fundamental cells can be identified with the projection of the monomials onto the red tiling plane. To reach the bulk of the cone (here the monomials $B$ and $C$ ), the path has to contain loops, e.g. small loops around a tiling node. For instance, the tip of the cone and $C$ are projected to the same point; therefore the corresponding path to $C$ must be a trivial loop. It can be chosen to be the appropriate power of any term in the superpotential. 
Corollary 5.3.3. For the consistency of the tiling a necessary condition is that homotopic paths of the same length are F-term equivalent. ${ }^{16}$

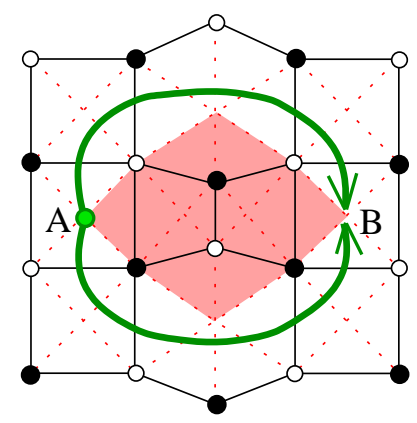

Figure 29: Inequivalent $A \rightarrow B$ homotopic paths in an inconsistent tiling.

If the tiling is inconsistent, it might be possible to construct two inequivalent paths surrounding the "inconsistency". An example is shown in Figure 29 where the tiling contains the subgraph of Figure 15 in [15]. We recognize the two rhombus paths and the corresponding tiling zig-zags along the boundary of the pink area. Since no F-terms can be used, the paths are inequivalent.

After proving the lemma and investigating some of its corollaries, let us turn back to the original problem. We want to show that the matrix element $S_{i j}$ gives the number of inequivalent paths from $i$ to $j$. In order to prove this, we need to show that for each $u$ lattice point in $\Delta_{i j}$, we have a unique allowed path in the tiling starting on the $i^{\text {th }}$ face and ending on the $j^{\text {th }}$ one. These $j^{\text {th }}$ faces are in different fundamental cells that are in one-to-one correspondence with the $u$ lattice points.

The previous lemma ensures that we have a single path for each cell. To see this, we need to prove that allowed homotopic paths have the same length. Suppose that there exist two homotopic paths of different lengths. Using F-term equations, we can deform the longer path to the shorter one as in Figure 30. Thus, we end up with loops around tiling nodes which are evidently not allowed, since these loops intersect $P M_{0}$. Recalling that F-terms transform allowed paths to allowed paths, we arrive at a contradiction. This means that in a consistent tiling homotopic allowed paths always have the same $R$-charge and are equivalent.

Having proved that from the $i^{\text {th }}$ face of a fixed fundamental cell there exists at most one inequivalent path to the $j^{\text {th }}$ face of any cell, we also need to show that these cells where the paths can end are in one-to-one correspondence with the $u$ lattice points. In order to do so, we reinterpret the (5.6) bounding inequalities of $\Delta_{i j}$.

In the definition of $\Delta_{i j}$, we have a $u \cdot v_{r} \leq a_{r}$ constraint for each external node of the toric diagram. For a given path, $u$ is interpreted as the integer vector defined on the lattice of fundamental

${ }^{16} \mathrm{An}$ immediate question arises: Is this condition sufficient? Can consistency be defined as the equivalence of homotopic paths? We leave this question for future study. 

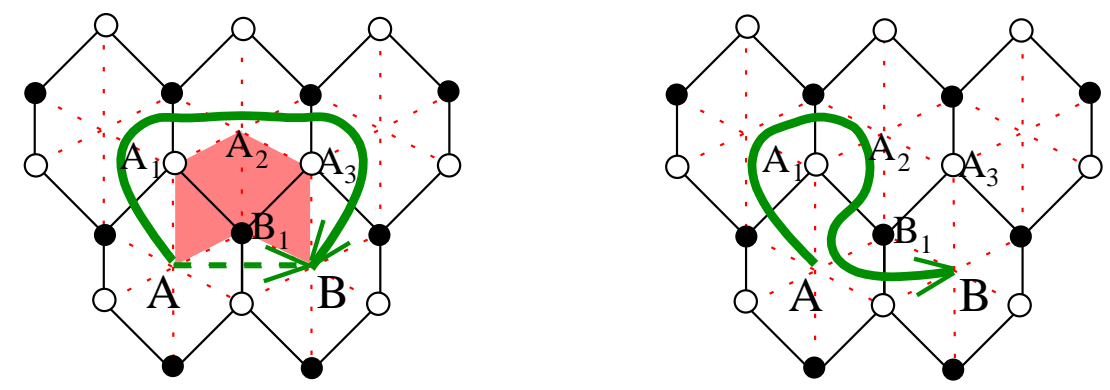

Figure 30: Homotopic paths with different $\mathrm{R}$-charge are not equivalent. After applying the $\mathrm{F}$-term equation for $A_{3} B_{1}$, the long path (solid green line) gets transformed to the short path (dashed line) plus a small loop around the $A_{1}$ node in the tiling.

cells giving the distance of the cells wherein the $i^{\text {th }}$ and $j^{\text {th }}$ faces reside. In the tiling language, $v_{r}$ is the monodromy of the height of the $r^{\text {th }}$ external perfect matching. Thus, the scalar product gives the increase in the $r^{\text {th }}$ height coordinate. Hence, the $a_{r}$ variables should be interpreted as height differences. In fact, this is exactly how we computed them with the $\Psi$-map in section 5.2 .

Figure 31 illustrates the correspondence schematically. The figure shows three inequivalent allowed paths that connect face $A$ to different $B$ faces. The shading indicates the $r^{\text {th }}$ height function. The height changes along the edges in the superposition of the corresponding matching and $P M_{0}$. This level set is represented by purple dashed lines.

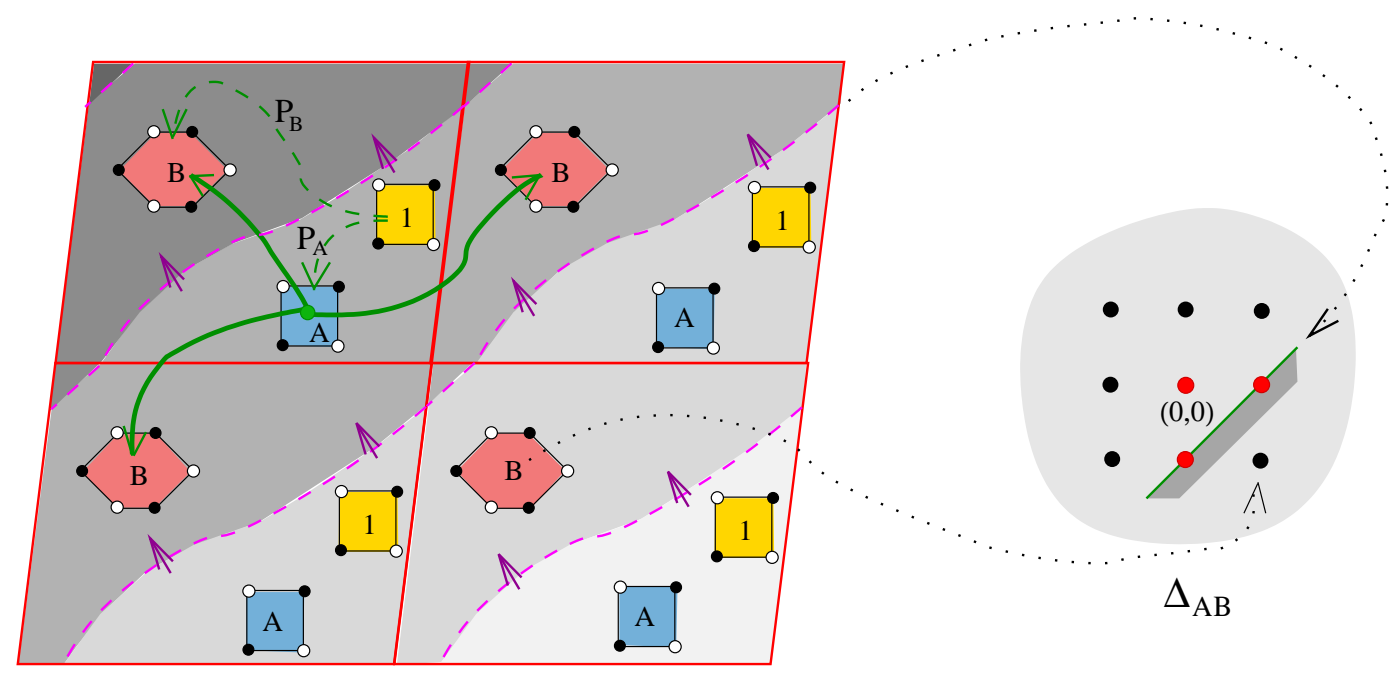

Figure 31: The figure schematically depicts three allowed green paths from $A$ to $B$. The shading indicates one of the height coordinates. The height increases in the direction of the small arrows. The allowed paths can only cross the dashed lines in this direction, and thus we obtain a bounding inequality for $\Delta_{A B}$. The remaining edges can be determined by means of the other heights. 
The right-hand side of Figure 31 shows the lattice of $\Delta_{A B}$ along with a green bounding line. The lattice points are in one-to-one correspondence with the red fundamental cells on the left-hand side. In particular, we assign them to the $B$ faces sitting in the cells. We set the origin at the middle point which is assigned to the upper left $B$ face in the tiling.

How does the green constraint come about? From previous discussions in section 5.1 we know that allowed paths can only go uphill on the height function. For example, in Figure 31 the paths can cross the dashed lines in the direction of the small arrows; therefore we can't reach the $B$ face in the lower right corner. This face corresponds to the excluded point on the right-hand side shown by the dotted arrow.

Using the above interpretation of $u$, we can immediately write down a necessary (and sufficient) condition for the allowed paths. In our schematic example, we have $v_{r}=(1,-1)$ which is the average "gradient vector" of the height function. Naively, the constraint translates to the following inequality for the allowed paths

$$
u \cdot v_{r} \leq 0
$$

This is not quite right, because the paths start from $A$ not $B$. One can take this into account by adding the difference in their height coordinates to the right-hand side

$$
u \cdot v_{r} \leq d_{r}
$$

By using the $P_{A}$ and $P_{B}$ reference paths that connect the first node of the Beilinson quiver to $A$ and $B$, one can see this difference is given by $d_{r}=\Psi_{r}\left(P_{B}\right)-\Psi_{r}\left(P_{A}\right) .{ }^{17}$ Let us denote the $i^{\text {th }}$ line bundle in the exceptional collection by $\left(a_{1}^{i}, a_{2}^{i}, \ldots, a_{n}^{i}\right)$. Recalling from section 5.2 how we have determined the collection, we obtain $d_{r}=a_{r}^{B}-a_{r}^{A}$. Our final expression is then

$$
u \cdot v_{r} \leq a_{r}^{B}-a_{r}^{A}
$$

which is precisely the inequality in the definition of $\Delta_{A B}$ !

We can write down the remaining inequalities for the constraints coming from the other height functions in exactly the same way. Thus, we obtain the boundaries of $\Delta_{A B}$.

We have seen that the inequalities are equivalent to the fact that allowed paths can't go downhill on any of the height functions of the external matchings. This completes the correspondence between the lattice points of $\Delta$ and the allowed paths, and thus proves that $S_{i j}$ indeed counts the inequivalent paths in the tiling.

Let us summarize the main results of this section. Given a consistent brane tiling, we can compute a $\mathcal{B}$ Beilinson quiver and an $\left\{E_{i}\right\}$ collection of line bundles by means of an internal matching and the $\Psi$-map. ${ }^{18}$ One may check on a case-by-case basis that this collection is exceptional.

\footnotetext{
${ }^{17}$ In the example of Figure 31, the difference is $d_{r}=1-0=1$, i.e. there is one level line between $A$ and $B$.

${ }^{18}$ For a specific Calabi-Yau, there are many equivalent Seiberg dual phases of the quiver theory [6, 54, 55, 56, 57, 58, 59, 10, 15. Notice that the exceptional collection of section 5.2 has the advantage that it gives back the right phase of the theory when computing the $S^{-1}$ quiver adjacency matrix.
} 
In this section we have proved that the "true" Beilinson quiver of the gauge theory living in the worldvolume of the D3-branes is the same as $\mathcal{B}$, the original quiver which is obtained directly from the tiling. In particular, we proved that the number of inequivalent paths between two nodes are the same.

As a byproduct, we obtained that homotopic paths with the same $\mathrm{R}$-charge are $\mathrm{F}$-term equivalent. Thus, we could clarify the relation of the brane tiling to the dual cone by a projection of the lattice points of the cone onto the tiling plane. This gave an explicit correspondence between monomials and paths.

\section{Conclusions}

Brane tilings can be deceptively simple. With a few strokes of a pen, all of the data of a $\mathcal{N}=1$ supersymmetric quiver gauge theory - the matter fields, the gauge groups, the superpotential - are captured. Given these simple pictures, theorems should be easy to prove, but we have often found otherwise. In the following paragraphs, we outline our successes but also the work that remains to be done to prove our dictionary between brane tilings and exceptional collections.

In section 4, we provided a recipe that will convert any exceptional collection of line bundles into a periodic quiver and motivated the recipe using Wilson lines and a little mirror symmetry. In the cases we looked at, this periodic quiver was the graph theoretic dual of a brane tiling. Thinking of the periodic quiver as a triangulation of a surface, we proved that the Euler character vanished. Since the exceptional collection specifies the connectivity of all the vertices, edges, and faces, a vanishing Euler character is not necessarily enough to ensure the quiver can be written on a torus. We hope to return to this issue in the future.

In section 5, we provided a recipe that will convert any brane tiling into a collection of line bundles. Two key observations underlie this recipe. The first is that internal perfect matchings of the tilling are in one-to-one correspondence with Beilinson quivers and hence with exceptional collections. The second is that external perfect matchings are in one-to-one correspondence with the generating Weil divisors $D_{r}$ and can be used to convert paths in the brane tiling into sums of divisors $\sum a_{r} D_{r}$ via the $\Psi$-map.

We left the word exceptional out of the first sentence of the preceding paragraph on purpose. On a case by case basis, we can verify the collections are exceptional, using for example the techniques described in 12]. However, proving that the collection is exceptional in general is difficult. There is a paper by Altmann and Hille [60] who prove strong exceptionality for quivers without relations (no superpotential) using Kodaira vanishing. The Kodaira vanishing theorem and certain generalizations are a powerful way of proving strong exceptionality. Given a line bundle $\mathcal{O}(D)$ corresponding to an ample divisor $D$, then

$$
\operatorname{dim} H^{q}(X, \mathcal{O}(D \otimes K))=0, \text { for any } q>0
$$


Unfortunately, for us, even in relatively simple exceptional collections, one finds a $D$ which is not ample even though these higher cohomology groups vanish. To see the vanishing, one must rely on techniques specific to the complex surface $V$ in question.

We hope the future brings new progress on both these fronts.

Acknowledgements: We gratefully acknowledge the invaluable discussions we have had with Aaron Bergman, Agostino Butti, Charles Doran, Sebastian Franco, Jonathan Heckman, Amer Iqbal, Robert Karp, Alastair King, David Morrison, Cumrun Vafa and Alberto Zaffaroni. We thank the KITP for hospitality during part of this work. AH would like to thank the string theory groups in Milan, Jerusalem, Valdivia and Buenos Aires for their kind hospitality while this work was being completed.

\section{Appendix}

To demonstrate the computation of exceptional collections with the $\Psi$-map of section 5.2, we give another example. This is the $Y^{3,2}$ theory, whose quiver is shown in Figure 32.

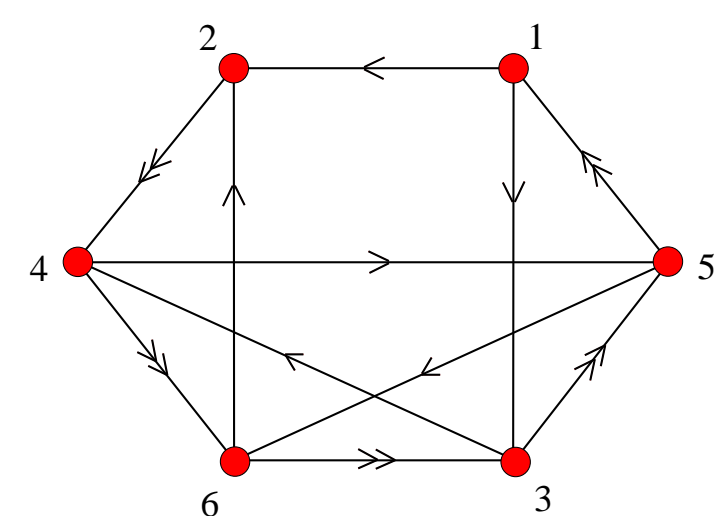

Figure 32: $Y^{3,2}$ quiver.

The brane tiling of this geometry and the 18 perfect matchings are given in Figure 33 and Figure 34. In the upper left corner of the figures the toric diagram is shown with a red dot giving the position of the matching. For reference matching we pick the $7^{\text {th }}$ matching of Figure 33 . Deleting the corresponding arrows in the quiver gives the Beilinson quiver (Figure 35). We need to fix allowed reference paths in the tiling that connect the first node of the Beilinson quiver to all the other nodes. The chosen paths are shown in Figure 36. 

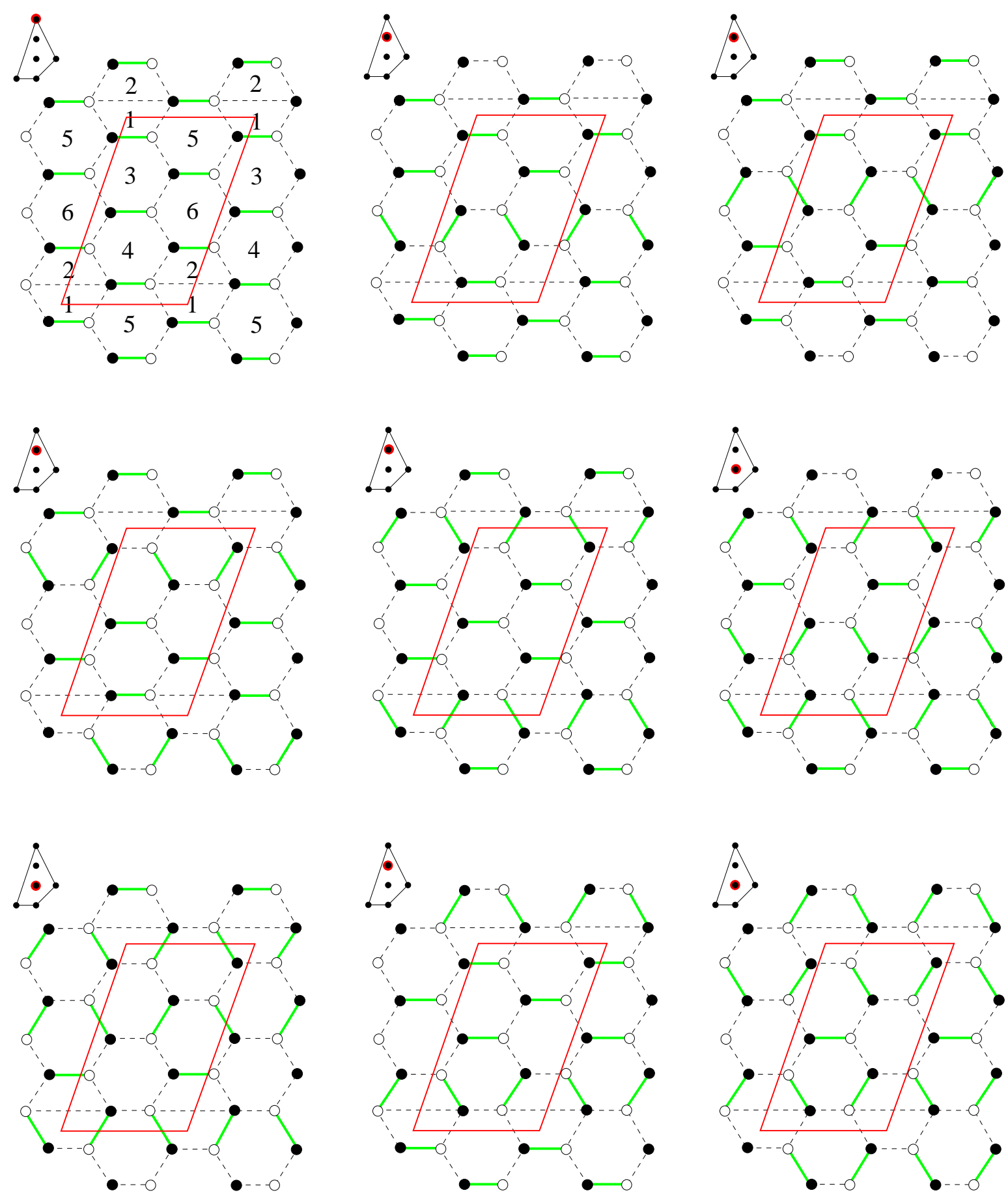

Figure 33: $Y^{3,2}$ perfect matchings $\left(1^{s t} \ldots 9^{t h}\right)$. 

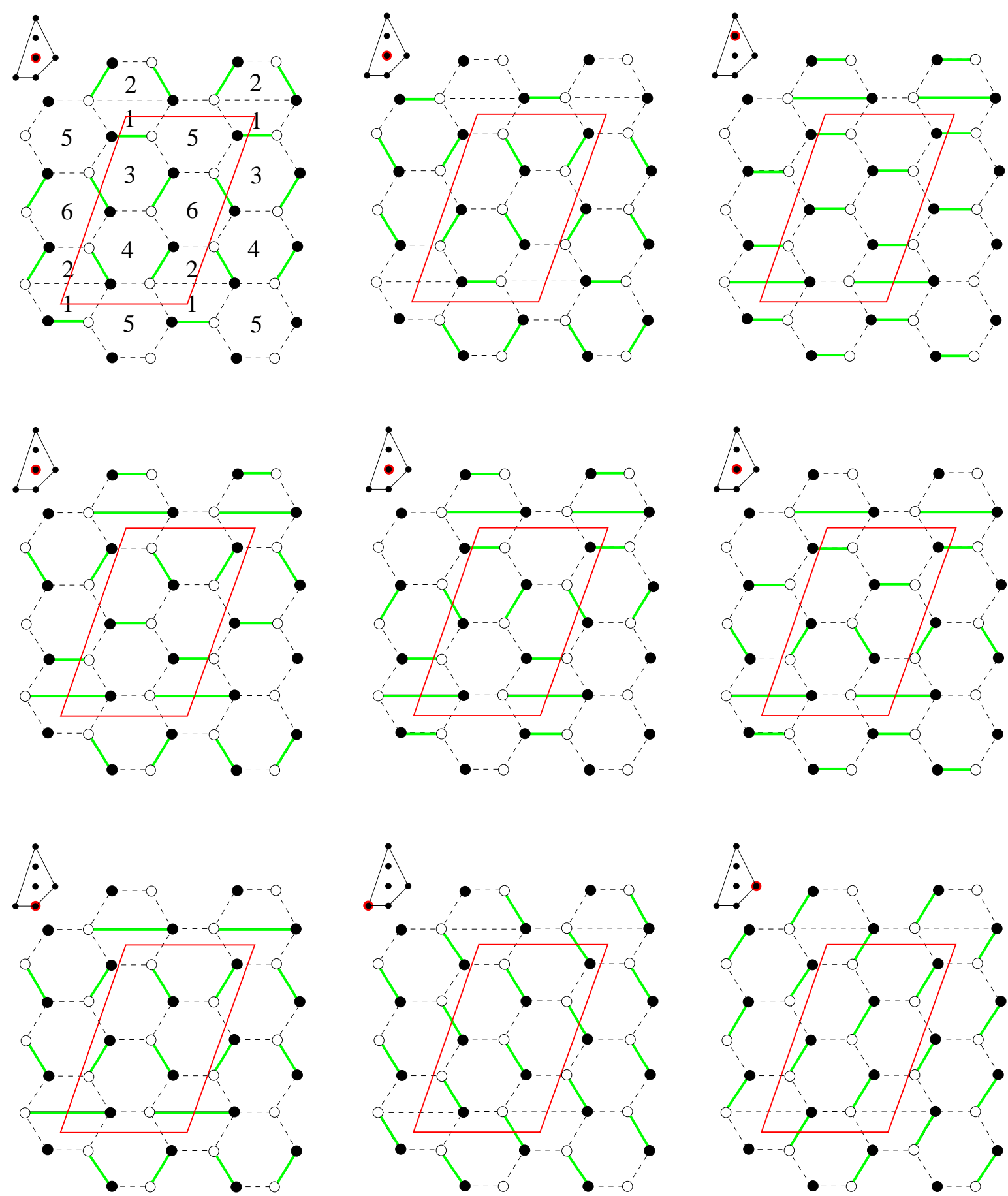

Figure 34: $Y^{3,2}$ perfect matchings $\left(10^{\text {th }} \ldots 18^{\text {th }}\right)$. 


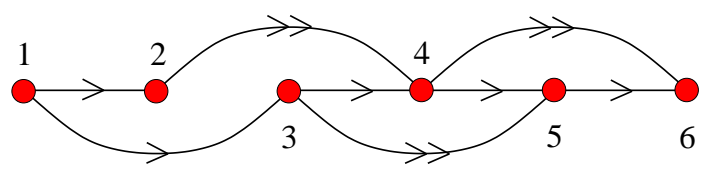

Figure 35: $Y^{3,2}$ Beilinson quiver. Bifundamentals in internal matching 7 are omitted.

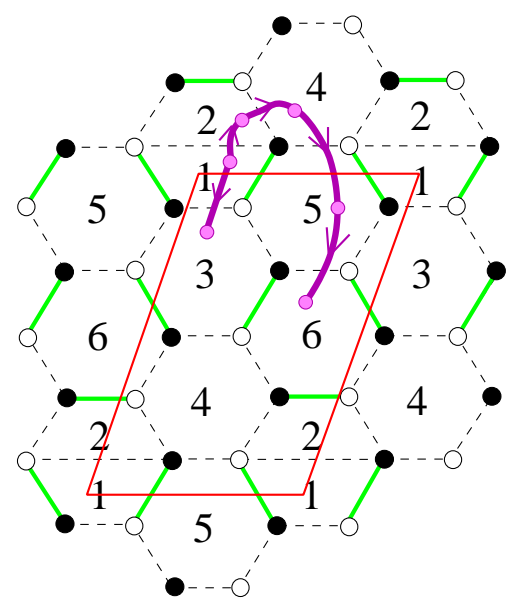

Figure 36: $Y^{3,2}$ tiling. The purple lines indicate the chosen paths that are used to compute the exceptional collections. The paths start on face 1 and connect it to the other faces.

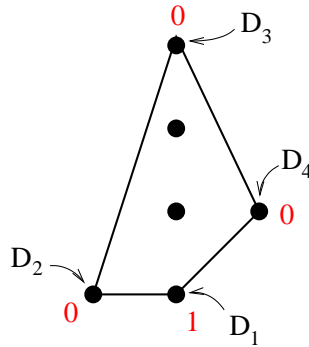

$1 \rightarrow 2$

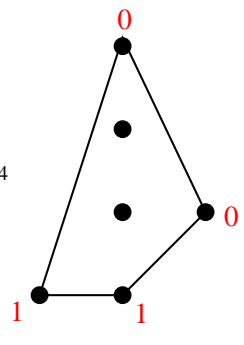

$1 \rightarrow 2 \rightarrow 4$

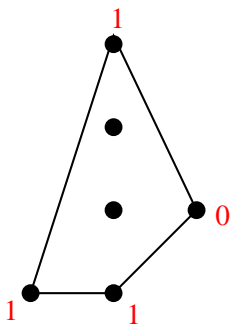

$1 \rightarrow 2 \rightarrow 4 \rightarrow 5 \quad 1 \rightarrow 2 \rightarrow 4 \rightarrow 5 \rightarrow 6$

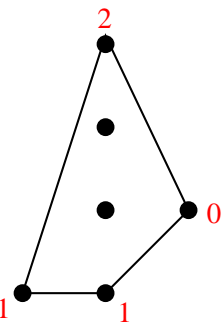

$1 \rightarrow 2 \rightarrow 4 \rightarrow 5 \rightarrow 6 \quad 1 \rightarrow 3$

Figure 37: A set of reference paths for $Y^{3,2}$.

From the intersection number of the paths and the external perfect matchings we can immediately derive the following collection:

$$
(0,0,0,0),(1,0,0,0),(0,0,1,0),(1,1,0,0),(1,1,1,0),(1,1,2,0) \text {. }
$$




\section{References}

[1] A. Hanany and K. D. Kennaway, Dimer models and toric diagrams, hep-th/0503149.

[2] S. Franco, A. Hanany, K. D. Kennaway, D. Vegh, and B. Wecht, Brane dimers and quiver gauge theories, hep-th/0504110.

[3] S. Franco, A. Hanany, D. Martelli, J. Sparks, D. Vegh, and B. Wecht, Gauge theories from toric geometry and brane tilings, hep-th/0505211.

[4] F. Cachazo, B. Fiol, K. A. Intriligator, S. Katz, and C. Vafa, A geometric unification of dualities, Nucl. Phys. B628 (2002) 3-78, hep-th/0110028.

[5] B. Feng, A. Hanany, and Y.-H. He, D-brane gauge theories from toric singularities and toric duality, Nucl. Phys. B595 (2001) 165-200, hep-th/0003085.

[6] B. Feng, A. Hanany, and Y.-H. He, Phase structure of D-brane gauge theories and toric duality, JHEP 08 (2001) 040, hep-th/0104259].

[7] M. Wijnholt, Large volume perspective on branes at singularities, Adv. Theor. Math. Phys. 7 (2004) 1117-1153, hep-th/0212021.

[8] P. S. Aspinwall and S. Katz, Computation of superpotentials for d-branes, hep-th/0412209.

[9] P. S. Aspinwall and L. M. Fidkowski, Superpotentials for quiver gauge theories, hep-th/0506041.

[10] C. P. Herzog, Seiberg duality is an exceptional mutation, JHEP 08 (2004) 064, hep-th/0405118.

[11] P. S. Aspinwall and I. V. Melnikov, D-branes on vanishing del pezzo surfaces, JHEP 12 (2004) 042, hep-th/0405134.

[12] C. P. Herzog and R. L. Karp, Exceptional collections and d-branes probing toric singularities, hep-th/0507175.

[13] A. Bergman and N. J. Proudfoot, Moduli spaces for d-branes at the tip of a cone, hep-th/0510158.

[14] C. P. Herzog and J. Walcher, Dibaryons from exceptional collections, JHEP 09 (2003) 060, hep-th/0306298.

[15] A. Hanany and D. Vegh, Quivers, tilings, branes and rhombi, hep-th/0511063.

[16] B. Feng, Y.-H. He, K. D. Kennaway, and C. Vafa, Dimer models from mirror symmetry and quivering amoebae, hep-th/0511287.

[17] M. R. Douglas and G. W. Moore, D-branes, quivers, and ale instantons, hep-th/9603167.

[18] M. R. Douglas, B. Fiol, and C. Romelsberger, The spectrum of bps branes on a noncompact calabi-yau, JHEP 09 (2005) 057, hep-th/0003263.

[19] A. Bergman and N. J. Proudfoot, Moduli spaces for bondal quivers, math.ag/0512166.

[20] N. C. Leung and C. Vafa, Branes and toric geometry, Adv. Theor. Math. Phys. 2 (1998) 91-118, hep-th/9711013.

[21] W. Fulton, Introduction to Toric Varieties. Princeton University Press, 1993. 
[22] D. Martelli and J. Sparks, Toric geometry, sasaki-einstein manifolds and a new infinite class of ads/cft duals, hep-th/0411238.

[23] D. Martelli, J. Sparks, and S. T. Yau, The geometric dual of a-maximisation for toric Sasaki- Einstein manifolds, hep-th/0503183.

[24] M. Cvetic, H. Lu, D. N. Page, and C. N. Pope, New einstein-sasaki spaces in five and higher dimensions, hep-th/0504225.

[25] M. Cvetic, H. Lu, D. N. Page, and C. N. Pope, New einstein-sasaki and einstein spaces from kerr-de sitter, hep-th/0505223.

[26] S. Benvenuti and M. Kruczenski, From sasaki-einstein spaces to quivers via bps geodesics: Lpqr, hep-th/0505206.

[27] A. Butti, D. Forcella, and A. Zaffaroni, The dual superconformal theory for lpqr manifolds, hep-th/0505220.

[28] A. Okounkov, N. Reshetikhin, and C. Vafa, Quantum Calabi-Yau and classical crystals, hep-th/0309208.

[29] A. Iqbal, N. Nekrasov, A. Okounkov, and C. Vafa, Quantum foam and topological strings, hep-th/0312022.

[30] A. Hanany and A. Zaffaroni, On the realization of chiral four-dimensional gauge theories using branes, JHEP 05 (1998) 001, hep-th/9801134.

[31] A. Hanany and A. M. Uranga, Brane boxes and branes on singularities, JHEP 05 (1998) 013, hep-th/9805139.

[32] M. Aganagic, A. Karch, D. Lust, and A. Miemiec, Mirror symmetries for brane configurations and branes at singularities, Nucl. Phys. B569 (2000) 277-302, hep-th/9903093.

[33] S. Franco and D. Vegh, Moduli spaces of gauge theories from dimer models: Proof of the correspondence, hep-th/0601063.

[34] R. Kenyon, An introduction to the dimer model, math.C0/0310326.

[35] R. Kenyon, A. Okounkov, and S. Sheffield, Dimers and amoebae, math-ph/0311005.

[36] A. Butti and A. Zaffaroni, R-charges from toric diagrams and the equivalence of a-maximization and z-minimization, JHEP 11 (2005) 019, hep-th/0506232.

[37] A. Butti and A. Zaffaroni, From toric geometry to quiver gauge theory: The equivalence of a-maximization and z-minimization, hep-th/0512240.

[38] P. Mayr, Phases of supersymmetric d-branes on kaehler manifolds and the mckay correspondence, JHEP 01 (2001) 018, hep-th/0010223.

[39] A. Tomasiello, D-branes on calabi-yau manifolds and helices, JHEP 02 (2001) 008, hep-th/0010217.

[40] S. Govindarajan and T. Jayaraman, D-branes, exceptional sheaves and quivers on calabi-yau manifolds: From mukai to mckay, Nucl. Phys. B600 (2001) 457-486, hep-th/0010196. 
[41] K. Hori, A. Iqbal, and C. Vafa, D-branes and mirror symmetry, hep-th/0005247.

[42] E. Zaslow, Solitons and helices: The search for a math physics bridge, Commun. Math. Phys. 175 (1996) 337-376, hep-th/9408133.

[43] M. Wijnholt, Parameter space of quiver gauge theories, hep-th/0512122.

[44] P. S. Aspinwall, D-branes, pi-stability and theta-stability, hep-th/0407123.

[45] J. Polchinski, String Theory. Cambridge University Press, 1998.

[46] V. Guillemin, Kaehler structures on toric varieties, J. Differential Geometry 40 (1994).

[47] M. Abreu, Kaehler geometry of toric manifolds in symplectic coordinates, math.DG/0004122.

[48] C. P. Herzog, Exceptional collections and del pezzo gauge theories, JHEP 04 (2004) 069, hep-th/0310262.

[49] H. Kihara, M. Sakaguchi, and Y. Yasui, Scalar laplacian on sasaki-einstein manifolds y(p,q), Phys. Lett. B621 (2005) 288-294, hep-th/0505259.

[50] T. Oota and Y. Yasui, Toric sasaki-einstein manifolds and heun equations, hep-th/0512124.

[51] S. Benvenuti, A. Hanany, D. Martelli, and J. Sparks, Work in progress, .

[52] S. Benvenuti and M. Kruczenski, Semiclassical strings in sasaki-einstein manifolds and long operators in $n=1$ gauge theories, hep-th/0505046.

[53] A. King and D. Vegh, Unpublished, .

[54] B. Feng, A. Hanany, Y.-H. He, and A. M. Uranga, Toric duality as Seiberg duality and brane diamonds, JHEP 12 (2001) 035, hep-th/0109063.

[55] B. Feng, S. Franco, A. Hanany, and Y.-H. He, Symmetries of toric duality, JHEP 12 (2002) 076, hep-th/0205144.

[56] D. Berenstein and M. R. Douglas, Seiberg duality for quiver gauge theories, hep-th/0207027.

[57] B. Feng, A. Hanany, Y. H. He, and A. Iqbal, Quiver theories, soliton spectra and picard-lefschetz transformations, JHEP 02 (2003) 056, hep-th/0206152].

[58] S. Franco, A. Hanany, and Y.-H. He, A trio of dualities: Walls, trees and cascades, Fortsch. Phys. 52 (2004) 540-547, hep-th/0312222.

[59] S. Benvenuti, A. Hanany, and P. Kazakopoulos, The toric phases of the $Y(p, q)$ quivers, hep-th/0412279.

[60] K. Altmann and L. Hille, Strong exceptional sequences provided by quivers, Algebras and Representation Theory 2 (1999) 1-17. 